\title{
ON THE KOTTWITZ-SHELSTAD NORMALIZATION OF TRANSFER FACTORS FOR AUTOMORPHIC INDUCTION FOR GL $\mathrm{GL}_{n}$
}

\author{
KAORU HIRAGA AND ATSUSHI ICHINO
}

\section{To the memory of Professor Hiroshi Saito}

\begin{abstract}
Automorphic induction for $\mathrm{GL}_{n}$ is a case of endoscopic transfer, and its character identity was established by Henniart and Herb, up to a constant of proportionality. We determine this constant in terms of the Kottwitz-Shelstad normalization of transfer factors, which involves certain $\varepsilon$-factors.
\end{abstract}

\section{Contents}

Introduction . . . . . . . . . . . . . . . . . . . . . . . 97

1. Statement of the main theorem . . . . . . . . . . . . . . . . . . . 99

2. Reduction to the standard $L$-group structures . . . . . . . . . . . . . . . . . . 102

3. Reduction to the standard Whittaker data . . . . . . . . . . . . . . . . 105

4. Reduction to Levi subgroups . . . . . . . . . . . . . . . . . . . . 107

5. Reduction to tamely ramified cyclic extensions . . . . . . . . . . . . . . . . . 112

6. Intertwining operators . . . . . . . . . . . . . . . . . . . . 115

7. Calculation of a twisted character . . . . . . . . . . . . . . . . 119

8. Calculation of a twisted orbital integral . . . . . . . . . . . . . . . 125

9. Calculation of the transfer factor . . . . . . . . . . . . . . . . . . . 128

10. Proof of Theorem 1.4 . . . . . . . . . . . . . . . . . . 136

References . . . . . . . . . . . . . . . . . . . 143

\section{Introduction}

Let $F$ be a nonarchimedean local field of characteristic zero. Let $\mathbf{G}=\mathrm{GL}_{n}$, and let

$$
\mathbf{H}=\operatorname{Res}_{E / F} \mathrm{GL}_{m_{1}} \times \cdots \times \operatorname{Res}_{E / F} \mathrm{GL}_{m_{r}}
$$

Received March 17, 2011. Revised September 8, 2011. Accepted May 2, 2012.

2010 Mathematics Subject Classification. Primary 22E50.

Hiraga's work was partially supported by Japan Society for the Promotion of Science Grant-in-Aid for Scientific Research (C) 22540018. Ichino's work was partially supported by Japan Society for the Promotion of Science Grant-in-Aid for Young Scientists (B) 22740021.

(C) 2012 by The Editorial Board of the Nagoya Mathematical Journal 
where $E$ is a cyclic extension of $F$ of degree $d$ and $\left(m_{1}+\cdots+m_{r}\right) d=n$. Then $\mathbf{H}$ is a twisted endoscopic group of $\mathbf{G}$. Let $G=\mathbf{G}(F)$, and let $H=\mathbf{H}(F)$. By a result of Waldspurger [20, corollaire 1.7], we have a map

$\operatorname{Tran}_{H}^{G}:\{$ virtual characters of $H\} \longrightarrow\{\omega$-twisted virtual characters of $G\}$

determined by matching orbital integrals. Here $\omega$ is a character of $F^{\times}$of order $d$ associated to $E / F$ by class field theory.

Automorphic induction for $\mathrm{GL}_{n}$ asserts that for each irreducible tempered admissible representation $\pi_{H}$ of $H$, there exist an irreducible tempered admissible representation $\pi$ of $G$ and a constant $c \in \mathbb{C}^{\times}$such that $\pi \otimes \omega \cong \pi$ and

$$
\operatorname{Tran}_{H}^{G}\left(\operatorname{trace}\left(\pi_{H}\right)\right)=c \cdot \operatorname{trace}\left(\pi \circ \mathcal{A}_{\omega}\right)
$$

Here $\mathcal{A}_{\omega}: \pi \otimes \omega \rightarrow \pi$ is an intertwining operator. This was established by Henniart and Herb [7] when $r=1$, and it is given by the composition of the parabolic induction from $H$ to $\mathrm{GL}_{m_{1}+\cdots+m_{r}}(E)$ with the automorphic induction from $\mathrm{GL}_{m_{1}+\cdots+m_{r}}(E)$ to $G$ in general. Henniart and Lemaire [8], $[9],[10]$ extended the fundamental lemma and the automorphic induction to the case of positive characteristic. Also, the automorphic induction has been extended to the case of inner forms of $\mathrm{GL}_{n}$ in [11].

The purpose of this paper is to determine the constant $c$ for automorphic induction for $\mathrm{GL}_{n}$ in the case of characteristic zero. To be precise, $\operatorname{Tran}_{H}^{G}$ depends on a transfer factor $\Delta$ which is well defined up to a scalar. Also, $\mathcal{A}_{\omega}$ is well defined up to a scalar. In particular, we first need to normalize $\Delta$ and $\mathcal{A}_{\omega}$ to determine the constant $c$. Following Kottwitz and Shelstad [13], we can take $\Delta$ and $\mathcal{A}_{\omega}$ which depend only on an $F$-splitting $\mathbf{s p l}_{G}$ of $\mathbf{G}$ and a nontrivial character $\psi$ of $F$ since $\mathbf{G}$ is quasi-split. Hence, $c$ is well defined.

For $n=2$, Labesse and Langlands [14] proved that $c=1$. For arbitrary $n$, Henniart and Lemaire [9] proved that $c$ does not depend on the representations and that $c=1$ if $E / F$ is unramified. In this paper, we will prove that

$$
c=1
$$

in general (see Theorem 1.4). We remark that the transfer factor $\Delta$ involves a certain $\varepsilon$-factor which depends on the twisted endoscopic group $\mathbf{H}$.

This paper is organized as follows. In Section 1, we recall the definition of $\operatorname{Tran}_{H}^{G}$ and state the main theorem. We prove standard reductions in Sections 2-4 and recall a result of Henniart and Lemaire [9] on the product 
formula for the constant $c$ in Section 5 . These imply that we may assume that $E / F$ is tamely ramified, $\mathbf{H}=\operatorname{Res}_{E / F} \mathbb{G}_{m}$, and $\pi_{H}=\mathbb{1}$. To prove that $c=1$ in this case, we will compute the two sides of (0.1) explicitly. In Section 6 , we construct the intertwining operator $\mathcal{A}_{\omega}$ by using a result of Shahidi [17]. In Section 7, we compute the twisted character trace $\left(\pi\left(f^{G}\right) \circ \mathcal{A}_{\omega}\right)$ for a certain $f^{G} \in C_{c}^{\infty}(G)$. Since $\pi_{H}=\mathbb{1}$, it remains to compute the twisted orbital integral for $f^{G}$ and the transfer factor $\Delta$. Following Waldspurger [19], we compute the twisted orbital integral and prove descent to topological unipotent elements in Section 8. In Section 9, we compute the transfer factor $\Delta$ explicitly. Using these and the fundamental lemma [19], we finish the proof of the main theorem in Section 10.

Notation. Let $F$ be a nonarchimedean local field of characteristic zero. We fix an algebraic closure $\bar{F}$ of $F$. Let $\Gamma=\operatorname{Gal}(\bar{F} / F)$ be the absolute Galois group of $F$, and let $W_{F}$ be the Weil group of $F$. Let $\mathfrak{o}=\mathfrak{o}_{F}$ be the maximal compact subring of $F$, let $\mathfrak{p}=\mathfrak{p}_{F}$ be the maximal ideal of $\mathfrak{o}$, let $\varpi=\varpi_{F}$ be a generator of $\mathfrak{p}$, and let $q=q_{F}$ be the cardinality of $\mathfrak{o} / \mathfrak{p}$. The valuation $\operatorname{ord}_{F}$ and the absolute value $|\cdot|$ on $F$ are normalized so that $\operatorname{ord}_{F} \varpi=1$ and $|\varpi|=q^{-1}$. We extend $|\cdot|$ to the absolute value on $\bar{F}$.

If $\mathbf{G}$ is a connected reductive algebraic group defined over $F$, let $G=$ $\mathbf{G}(F)$ be the group of rational points of $\mathbf{G}$, let $\mathbf{G}_{\mathrm{SC}}$ be the simply connected cover of the derived group of $\mathbf{G}$, and let $\hat{G}$ be the Langlands dual group of $\mathbf{G}$. If $\mathbf{T}$ is a maximal torus of $\mathbf{G}$, let $\mathbf{T}_{\mathrm{sc}}$ be the preimage of $\mathbf{T}$ in $\mathbf{G}_{\mathrm{SC}}$, and let

$$
D_{G}(\gamma)=\prod_{\alpha \in R(\mathbf{G}, \mathbf{T})}|\alpha(\gamma)-1|^{1 / 2}
$$

for $\gamma \in T$, where $R(\mathbf{G}, \mathbf{T})$ is the set of roots of $\mathbf{T}$ in $\mathbf{G}$.

\section{$\S 1$. Statement of the main theorem}

Let $\mathbf{G}=\mathrm{GL}_{n}$. Let $\mathbf{s p l}_{G}=\left(\mathbf{B}_{0}, \mathbf{T}_{0},\left\{X_{\alpha}\right\}\right)$ be an $F$-splitting of $\mathbf{G}$, and let $\psi$ be a nontrivial character of $F$. Then $\mathbf{s p l}_{G}$ and $\psi$ determine a character $\chi$ of $U_{0}$, where $\mathbf{U}_{0}$ is the unipotent radical of $\mathbf{B}_{0}$ and

$$
\chi\left(\exp \left(x X_{\alpha}\right)\right)=\psi(x)
$$

for $x \in F$ and a simple root $\alpha$ of $\mathbf{T}_{0}$ in $\mathbf{B}_{0}$. We call $\left(\mathbf{B}_{0}, \chi\right)$ a Whittaker datum for $\mathbf{G}$.

Let $\mathbf{a} \in \mathrm{H}^{1}\left(W_{F}, Z(\hat{G})\right)$, where $Z(\hat{G})$ is the center of $\hat{G}$. Let $(\mathbf{H}, \mathcal{H}, s, \xi)$ be an endoscopic datum for $(\mathbf{G}, 1, \mathbf{a})$ (see [13, Section 2.1]). 
Since $\mathbf{G}$ is quasi-split over $F$, we have the absolute transfer factor $\Delta_{0}$ (see [15, Section 3.7], [13, Section 5.3]). Recall that $\Delta_{0}$ is a function on

$$
\begin{aligned}
& \{G \text {-regular semisimple elements in } H\} \\
& \quad \times\{\text { regular semisimple elements in } G\}
\end{aligned}
$$

given by the product of five terms:

$$
\Delta_{0}=\Delta_{\mathrm{I}} \cdot \Delta_{\mathrm{II}} \cdot \Delta_{\mathrm{III}_{1}} \cdot \Delta_{\mathrm{III}_{2}} \cdot \Delta_{\mathrm{IV}} \cdot
$$

To be precise, we implicitly fix an $F$-splitting $\mathbf{s p l}_{H}=\left(\mathbf{B}_{H, 0}, \mathbf{T}_{H, 0},\left\{Y_{\alpha}\right\}\right)$ of $\mathbf{H}$ and a $\Gamma$-splitting $\mathbf{s p l}_{\hat{G}}=\left(\mathcal{B}, \mathcal{T},\left\{\mathcal{X}_{\check{\alpha}}\right\}\right)$ (resp., $\left.\mathbf{s p l}_{\hat{H}}=\left(\mathcal{B}_{H}, \mathcal{T}_{H},\left\{\mathcal{Y}_{\check{\alpha}}\right\}\right)\right)$ of $\hat{G}$ (resp., $\hat{H})$. Moreover, by replacing $(\mathbf{H}, \mathcal{H}, s, \xi)$ by an endoscopic datum $(\mathbf{H}, \mathcal{H}, \operatorname{Int}(g)(s), \operatorname{Int}(g) \circ \xi)$ with some $g \in \hat{G}$ if necessary, we assume that $\xi\left(\mathcal{B}_{H}\right) \subset \mathcal{B}$ and that $\xi\left(\mathcal{T}_{H}\right)=\mathcal{T}$. However, $\Delta_{0}$ depends only on $\mathbf{s p l}_{G}$. Following [13, Section 5.3], we define a normalized transfer factor $\Delta=\Delta_{G, H}$ by

$$
\Delta=\varepsilon(V, \psi) \cdot \Delta_{0}
$$

where

$$
V=X^{*}\left(\mathbf{T}_{0}\right) \otimes \mathbb{C}-X^{*}\left(\mathbf{T}_{H, 0}\right) \otimes \mathbb{C}
$$

is a virtual representation of $\Gamma$ of dimension zero and $\varepsilon(V, \psi)$ is the local constant as in $[18,(3.6)]$. Then $\Delta$ depends only on the Whittaker datum $\left(\mathbf{B}_{0}, \chi\right)$ associated to $\mathbf{s p l}_{G}$ and $\psi$.

Remark 1.1. By [11, Proposition A.2], the transfer factor given by Henniart and Herb [7] agrees with $\Delta$ if $E / F$ is unramified, and it agrees with $\Delta$ up to a scalar in general.

Let $\omega$ be the character of $F^{\times}$associated to a. We write $\omega(g)=\omega(\operatorname{det} g)$ for $g \in G$. Fix Haar measures $d g$ and $d h$ on $G$ and $H$, respectively. For a regular semisimple element $\gamma \in G$ such that $G_{\gamma} \subset \operatorname{ker} \omega$ and $f^{G} \in C_{c}^{\infty}(G)$, put

$$
J^{\omega}\left(\gamma, f^{G}\right)=\int_{G_{\gamma} \backslash G} \omega(g) f^{G}\left(g^{-1} \gamma g\right) \frac{d g}{d t},
$$

where $\mathbf{G}_{\gamma}$ is the centralizer of $\gamma$ in $\mathbf{G}$ and $d t$ is a Haar measure on $G_{\gamma}$. Similarly, for a $G$-regular semisimple element $\gamma_{H} \in H$ and $f^{H} \in C_{c}^{\infty}(H)$, put

$$
J\left(\gamma_{H}, f^{H}\right)=\int_{H_{\gamma_{H}} \backslash H} f^{H}\left(h^{-1} \gamma_{H} h\right) \frac{d h}{d u},
$$


where $\mathbf{H}_{\gamma_{H}}$ is the centralizer of $\gamma_{H}$ in $\mathbf{H}$ and $d u$ is a Haar measure on $H_{\gamma_{H}}$. If $\gamma_{H}$ is a norm of $\gamma$, then we have $H_{\gamma_{H}} \cong G_{\gamma} \subset \operatorname{ker} \omega$, and we take compatible measures $d t$ and $d u$.

By a result of Waldspurger [20, corollaire 1.7], for each $f^{G} \in C_{c}^{\infty}(G)$ there exists $f^{H} \in C_{c}^{\infty}(H)$ such that

$$
J\left(\gamma_{H}, f^{H}\right)=\sum_{\gamma} \Delta\left(\gamma_{H}, \gamma\right) J^{\omega}\left(\gamma, f^{G}\right)
$$

for all $G$-regular semisimple elements $\gamma_{H} \in H$. Here the sum is taken over a set of representatives for the conjugacy classes of $\gamma \in G$ whose norm is $\gamma_{H}$. We say that $f^{G}$ and $f^{H}$ have matching orbital integrals. For a virtual character $\theta$ of $H$, we define an $\omega$-invariant distribution $\operatorname{Tran}_{H}^{G}(\theta)$ on $G$ by

$$
\operatorname{Tran}_{H}^{G}(\theta)\left(f^{G}\right)=\theta\left(f^{H}\right)
$$

for $f^{G} \in C_{c}^{\infty}(G)$. Here we say that a distribution $D$ on $G$ is $\omega$-invariant if

$$
D\left(\operatorname{Ad}(g)\left(f^{G}\right)\right)=\omega(g)^{-1} D\left(f^{G}\right)
$$

for all $g \in G$ and $f^{G} \in C_{c}^{\infty}(G)$.

On the other hand, by a result of Henniart and Herb [7, Theorem 1.3], for each irreducible tempered admissible representation $\pi_{H}$ of $H$ there exist an irreducible tempered admissible representation $\pi$ of $G$ on a space $V_{\pi}$ and a constant $c \in \mathbb{C}^{\times}$such that $\pi \otimes \omega \cong \pi$ and such that

$$
\operatorname{Tran}_{H}^{G}\left(J\left(\pi_{H}\right)\right)=c \cdot J^{\omega}(\pi) .
$$

Here $J\left(\pi_{H}, f^{H}\right)=\operatorname{trace}\left(\pi_{H}\left(f^{H}\right)\right)$ for $f^{H} \in C_{c}^{\infty}(H)$, and $J^{\omega}\left(\pi, f^{G}\right)=$ $\operatorname{trace}\left(\pi\left(f^{G}\right) \circ \mathcal{A}_{\omega}\right)$ for $f^{G} \in C_{c}^{\infty}(G)$, where $\mathcal{A}_{\omega}: V_{\pi} \rightarrow V_{\pi}$ is an isomorphism such that

$$
\mathcal{A}_{\omega} \circ(\pi \otimes \omega)(g)=\pi(g) \circ \mathcal{A}_{\omega}
$$

for all $g \in G$. We remark that $J^{\omega}(\pi)$ and $J\left(\pi_{H}\right)$ depend on $d g$ and $d h$, but the constant $c$ does not depend on the choice of Haar measures. Also, $J^{\omega}(\pi)$ depends on the choice of $\mathcal{A}_{\omega}$. Since $\pi$ is generic, we can normalize $\mathcal{A}_{\omega}$ so that

$$
\lambda \circ \mathcal{A}_{\omega}=\lambda,
$$

where $\lambda: V_{\pi} \rightarrow \mathbb{C}$ is a Whittaker functional with respect to $\chi$.

We now recall a result of Henniart and Lemaire [9]. 
THEOREM 1.2 [9, théorème 4.11]. The constant $c$ does not depend on $\pi_{H}$. Moreover, $c=1$ if $E / F$ is unramified.

REMARK 1.3. Henniart [6, théorème 2] proved an analogous result for $F=\mathbb{R}$.

Our main result is as follows.

THEOREM 1.4. We have

$$
c=1 \text {. }
$$

Remark 1.5. Arthur and Clozel [1] proved an analogous result for base change for $\mathrm{GL}_{n}$. Also, assuming certain properties of tempered $L$-packets, Waldspurger [21, lemme 4.8] proved an analogous result for special orthogonal groups.

\section{$\S 2$. Reduction to the standard $L$-group structures}

We retain the notation of Section 1 . Let $(\mathbf{H}, \mathcal{H}, s, \xi)$ be an endoscopic datum for $(\mathbf{G}, 1, \mathbf{a})$.

Let $\gamma \in G$ be a regular semisimple element. Let $\gamma_{H} \in H$ be a $G$-regular semisimple element. Assume that $\gamma_{H}$ is the image of $\gamma$. Let $\mathbf{T}$ (resp., $\mathbf{T}_{H}$ ) be the centralizer of $\gamma$ (resp., $\gamma_{H}$ ) in $\mathbf{G}$ (resp., $\mathbf{H}$ ). Let $i_{0}: \mathbf{T}_{H, 0} \rightarrow \mathbf{T}_{0}$ be the dual of $\xi^{-1}: \mathcal{T} \rightarrow \mathcal{T}_{H}$. We choose an admissible embedding

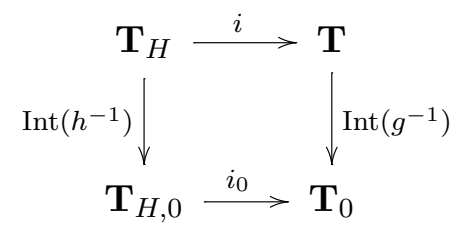

such that $i\left(\gamma_{H}\right)=\gamma$, where $h \in \mathbf{H}_{\mathrm{SC}}(\bar{F})$ and $g \in \mathbf{G}_{\mathrm{SC}}(\bar{F})$.

Let $\Delta$ be the transfer factor associated to $(\mathbf{H}, \mathcal{H}, s, \xi)$.

LEMMA 2.1. If $\Delta^{\prime}$ is the transfer factor associated to an endoscopic datum $\left(\mathbf{H}, \mathcal{H}, s^{\prime}, \xi\right)$ with $s^{\prime} \in \hat{G}$, then

$$
\Delta^{\prime}=\Delta
$$

Proof. By [11, Section 10], we have

$$
s^{\prime} \in s \cdot\left(Z(\hat{H})^{\Gamma}\right)^{0} .
$$

Hence, the image of $s^{\prime}$ in $\pi_{0}\left(\hat{T}_{\mathrm{ad}}^{\Gamma}\right)$ is equal to that of $s$. This completes the proof. 
Recall that $\mathcal{H}$ is a split extension

$$
1 \longrightarrow \hat{H} \longrightarrow \mathcal{H} \longrightarrow W_{F} \longrightarrow 1 \text {. }
$$

Let ${ }^{L} H=\hat{H} \rtimes W_{F}$ denote the $L$-group of $\mathbf{H}$ with an action of $\Gamma$ on $\hat{H}$ determined by $\mathbf{s p l}_{\hat{H}}$. Since the derived group of $\mathbf{H}$ is simply connected, the identity map $\hat{H} \rightarrow \hat{H}$ gives rise to an $L$-isomorphism $\mathcal{H} \rightarrow{ }^{L} H$ (see $[13$, Lemma 2.2.A]). This isomorphism is well defined up to a 1-cocycle of $W_{F}$ in $Z(\hat{H})$.

We now take $\mathcal{H}={ }^{L} H$. If $b$ is a 1 -cocycle of $W_{F}$ in $Z(\hat{H})$, let $\omega_{b}$ be the character of $H$ associated to $b$. Let $\xi_{b}:{ }^{L} H \hookrightarrow{ }^{L} G$ be the $L$-embedding given by

$$
\xi_{b}(h \times w)=\xi(b(w) h \times w)
$$

for $h \in \hat{H}$ and $w \in W_{F}$. Let $\Delta_{b}$ be the transfer factor associated to an endoscopic datum $\left(\mathbf{H},{ }^{L} H, s, \xi_{b}\right)$. Let $c_{b}$ be the constant for $\left(\mathbf{H},{ }^{L} H, s, \xi_{b}\right)$ given by (1.2).

LEMMA 2.2. We have

$$
\Delta_{b}\left(\gamma_{H}, \gamma\right)=\omega_{b}\left(\gamma_{H}\right) \cdot \Delta\left(\gamma_{H}, \gamma\right)
$$

Proof. It is easy to see that $\Delta_{\mathrm{I}}, \Delta_{\mathrm{II}}, \Delta_{\mathrm{III}_{1}}, \Delta_{\mathrm{IV}}$, and $\varepsilon(V, \psi)$ do not depend on $b$. Let $\xi_{T}:{ }^{L} T \rightarrow{ }^{L} G$, and let $\xi_{T_{H}}:{ }^{L} T_{H} \rightarrow{ }^{L} H$ be as in [15, Section 2.6]. We use the same $\chi$-data to compute the transfer factor $\Delta_{b}$. In particular, $\xi_{T}$ and $\xi_{T_{H}}$ do not depend on $b$. Let $\xi \circ \xi_{T_{H}}=a \cdot \xi_{T}$, where $a$ is a 1-cocycle of $W_{F}$ in $\mathcal{T}$. Then we have

$$
\xi_{b} \circ \xi_{T_{H}}=(\xi \circ b) \cdot a \cdot \xi_{T}
$$

Thus, we obtain

$$
\Delta_{\mathrm{III}_{2}, b}\left(\gamma_{H}, \gamma\right)=\omega_{b}\left(\gamma_{H}\right) \cdot \Delta_{\mathrm{III}_{2}}\left(\gamma_{H}, \gamma\right)
$$

This completes the proof.

Lemma 2.3. The constant $c_{b}$ does not depend on $b$.

Proof. Let $\phi_{H}$ be a tempered Langlands parameter for $\mathbf{H}$. Put $\phi_{H, b}=$ $b^{-1} \cdot \phi_{H}$. Let $\pi_{H}$ (resp., $\left.\pi_{H, b}\right)$ be the irreducible tempered admissible representation of $H$ associated to $\phi_{H}$ (resp., $\phi_{H, b}$ ). By [3, Lemma VII.2.1], we have

$$
J\left(\pi_{H, b}, \gamma_{H}\right)=\omega_{b}\left(\gamma_{H}\right)^{-1} \cdot J\left(\pi_{H}, \gamma_{H}\right)
$$


Hence, we have

$$
\operatorname{Tran}_{H, b}^{G}\left(J\left(\pi_{H, b}\right)\right)=\operatorname{Tran}_{H}^{G}\left(J\left(\pi_{H}\right)\right)
$$

by Lemma 2.2. Here $\operatorname{Tran}_{H, b}^{G}$ is the transfer for $\left(\mathbf{H},{ }^{L} H, s, \xi_{b}\right)$. Since $\xi_{b} \circ$ $\phi_{H, b}=\xi \circ \phi_{H}$, the assertion follows.

Let $\mathbf{m}=\left(m_{1}, \ldots, m_{r}\right)$ be a partition of $n / d$, and let $\left(\mathbf{H}_{\mathbf{m}},{ }^{L} H_{\mathbf{m}}, s_{\mathbf{m}}, \xi_{\mathbf{m}}\right)$ be an endoscopic datum for $(\mathbf{G}, 1, \mathbf{a})$ defined in [11, Section 10]. To prove Theorem 1.4 , we may assume that $(\mathbf{H}, \mathcal{H}, s, \xi)=\left(\mathbf{H}_{\mathbf{m}},{ }^{L} H_{\mathbf{m}}, s_{\mathbf{m}}, \xi_{\mathbf{m}}\right)$ by Lemmas 2.1 and 2.3 .

For convenience, we recall the definition of $\left(\mathbf{H}_{\mathbf{m}},{ }^{L} H_{\mathbf{m}}, s_{\mathbf{m}}, \xi_{\mathbf{m}}\right)$. Let $\zeta=$ $e^{2 \pi \sqrt{-1} / n}$. Let $a$ be the unique 1-cocycle of $W_{F}$ in $Z(\hat{G})$ representing a. Let $E$ be the cyclic extension of $F$ of degree $d$ associated to ker $a$ by class field theory. Let $\sigma$ be the generator of $\operatorname{Gal}(E / F)$ such that $a(\sigma)=\zeta^{n / d}$. We fix an element $w_{\sigma} \in W_{F}$ in the preimage of $\sigma$.

For each integer $m$, let $\mathbf{H}_{m}=\operatorname{Res}_{E / F} \mathrm{GL}_{m}$, let $\hat{H}_{m}=\mathrm{GL}_{m}(\mathbb{C})^{d}$, and let ${ }^{L} H_{m}=\hat{H}_{m} \rtimes W_{F}$. Here $W_{E}$ acts on $\hat{H}_{m}$ trivially, and $\sigma$ acts on $\hat{H}_{m}$ by

$$
\left(g_{1}, \ldots, g_{d}\right) \longmapsto\left(g_{d}, g_{1}, \ldots, g_{d-1}\right) .
$$

We take the standard splittings $\mathbf{s p l}_{H_{m}}$ and $\mathbf{s p l} \hat{H}_{m}$ of $\mathbf{H}_{m}$ and $\hat{H}_{m}$, respectively. For $x \in \mathbb{C}^{\times}$, put

$$
s_{m}(x)=\operatorname{diag}\left(x \cdot \mathbf{1}_{m}, \zeta^{n / d} x \cdot \mathbf{1}_{m}, \ldots, \zeta^{(d-1) n / d} x \cdot \mathbf{1}_{m}\right) \in \mathrm{GL}_{m d}(\mathbb{C}) .
$$

We define a homomorphism $\xi_{m, 0}:{ }^{L} H_{m} \rightarrow \mathrm{GL}_{m d}(\mathbb{C})$ by

$$
\xi_{m, 0}\left(\left(g_{1}, \ldots, g_{d}\right) \times w\right)=\operatorname{diag}\left(g_{1}, \ldots, g_{d}\right)
$$

for $\left(g_{1}, \ldots, g_{d}\right) \in \hat{H}_{m}$ and $w \in W_{E}$, and

$$
\xi_{m, 0}\left(1 \times w_{\sigma}\right)=\left(\begin{array}{cccc} 
& & & \mathbf{1}_{m} \\
\mathbf{1}_{m} & & & \\
& \ddots & & \\
& & \mathbf{1}_{m}
\end{array}\right) \text {. }
$$

Let $\mathbf{H}_{\mathbf{m}}=\mathbf{H}_{m_{1}} \times \cdots \times \mathbf{H}_{m_{r}}$, let $\hat{H}_{\mathbf{m}}=\hat{H}_{m_{1}} \times \cdots \times \hat{H}_{m_{r}}$, and let ${ }^{L} H_{\mathbf{m}}=$ $\hat{H}_{\mathbf{m}} \rtimes W_{F}$. Put

$$
s_{\mathbf{m}}=\operatorname{diag}\left(s_{m_{1}}\left(x_{1}\right), \ldots, s_{m_{r}}\left(x_{r}\right)\right) \in \mathrm{GL}_{n}(\mathbb{C}),
$$


where we choose $x_{1}, \ldots, x_{r} \in \mathbb{C}^{\times}$such that

$$
\left\{x_{i}, \zeta^{n / d} x_{i}, \ldots, \zeta^{(d-1) n / d} x_{i}\right\} \cap\left\{x_{j}, \zeta^{n / d} x_{j}, \ldots, \zeta^{(d-1) n / d} x_{j}\right\}=\varnothing
$$

if $i \neq j$. We define a homomorphism $\xi_{\mathbf{m}}:{ }^{L} H_{\mathbf{m}} \rightarrow{ }^{L} G$ by

$$
\xi_{\mathbf{m}}\left(\left(h_{1}, \ldots, h_{r}\right) \times w\right)=\operatorname{diag}\left(\xi_{m_{1}, 0}\left(h_{1} \times w\right), \ldots, \xi_{m_{r}, 0}\left(h_{r} \times w\right)\right) \times w
$$

for $\left(h_{1}, \ldots, h_{r}\right) \in \hat{H}_{\mathbf{m}}$ and $w \in W_{F}$. Note that the equivalence class of $\left(\mathbf{H}_{\mathbf{m}}\right.$, $\left.{ }^{L} H_{\mathbf{m}}, s_{\mathbf{m}}, \xi_{\mathbf{m}}\right)$ does not depend on the choice of $x_{1}, \ldots, x_{r}$.

\section{§3. Reduction to the standard Whittaker data}

We retain the notation of Section 1 . Let $\left(\mathbf{B}_{0}, \chi\right)$ be the Whittaker datum for $\mathbf{G}$ associated to $\mathbf{s p l}_{G}$ and $\psi$, where $\mathbf{s p l}_{G}$ is an $F$-splitting of $\mathbf{G}$ and $\psi$ is a nontrivial character of $F$. Let $\mathbf{m}$ be a partition of $n / d$, and let $(\mathbf{H}, \mathcal{H}, s, \xi)=$ $\left(\mathbf{H}_{\mathbf{m}},{ }^{L} H_{\mathbf{m}}, s_{\mathbf{m}}, \xi_{\mathbf{m}}\right)$ be the endoscopic datum for $(\mathbf{G}, 1, \mathbf{a})$ defined in Section 2 .

Let $\tilde{t} \in T_{0}$, and let $\mathbf{s p l}_{G}^{\prime}=\operatorname{Int}(\tilde{t})\left(\mathbf{s p l}_{G}\right)$. Let $\Delta^{\prime}$ be the transfer factor with respect to $\mathbf{s p l}_{G}^{\prime}$ and $\psi$.

LEMMA 3.1. We have

$$
\Delta^{\prime}=\omega(\operatorname{det} \tilde{t}) \cdot \Delta
$$

Proof. By replacing $\mathbf{s p l}_{G}$ by its $G_{\mathrm{SC}}$-conjugate if necessary, we may assume that $\mathbf{s p l}_{G}$ is the standard splitting of $\mathbf{G}$. Let $\mathbf{T}_{H}$ be a maximal torus of $\mathbf{H}_{\mathbf{m}}$ which is maximally split. We choose an admissible embedding $\mathbf{T}_{H} \rightarrow \mathbf{G}$, and let $\mathbf{T}$ be its image. We identify $\mathbf{T}_{H}$ with $\mathbf{T}$. Then we have an exact sequence

$$
1 \longrightarrow \mathbf{T}_{\mathrm{sc}} \longrightarrow \mathbf{T} \stackrel{\operatorname{det}}{\longrightarrow} \mathbb{G}_{m} \longrightarrow 1
$$

and its dual

$$
1 \longleftarrow \hat{T}_{\text {ad }} \longleftarrow \hat{T} \longleftarrow \mathbb{C}^{\times} \longleftarrow 1
$$

These induce long exact sequences

$$
\cdots \longrightarrow \mathrm{H}^{0}(F, \mathbf{T}) \longrightarrow \mathrm{H}^{0}\left(F, \mathbb{G}_{m}\right) \stackrel{\delta}{\longrightarrow} \mathrm{H}^{1}\left(F, \mathbf{T}_{\mathrm{sc}}\right) \longrightarrow \mathrm{H}^{1}(F, \mathbf{T}) \longrightarrow \cdots
$$

and

$$
\begin{aligned}
\cdots & \longleftarrow \mathrm{H}^{1}\left(W_{F}, \hat{T}\right) \longleftarrow \mathrm{H}^{1}\left(W_{F}, \mathbb{C}^{\times}\right) \stackrel{\hat{\delta}}{\longleftarrow} \mathrm{H}^{0}\left(W_{F}, \hat{T}_{\mathrm{ad}}\right) \\
& \longleftarrow \mathrm{H}^{0}\left(W_{F}, \hat{T}\right) \longleftarrow \cdots
\end{aligned}
$$


It is easy to see that

$$
\hat{\delta}\left(\text { the image of } s_{\mathbf{m}} \text { in } \hat{T}_{\mathrm{ad}}\right)=\mathbf{a} .
$$

Hence, we have

$$
\left\langle\delta(x), s_{\mathbf{m}}\right\rangle=\omega(x)
$$

for $x \in F^{\times}$, where

$$
\langle\cdot, \cdot\rangle: \mathrm{H}^{1}\left(F, \mathbf{T}_{\mathrm{sc}}\right) \times \pi_{0}\left(\hat{T}_{\mathrm{ad}}^{\Gamma}\right) \longrightarrow \mathbb{C}^{\times}
$$

is the Tate-Nakayama pairing.

For $\tilde{g} \in G$, let $g=z \cdot \tilde{g} \in \mathbf{G}_{\mathrm{SC}}(\bar{F})$, where we choose $z \in \bar{F}^{\times}$such that $\operatorname{det} \tilde{g}=z^{-n}$. Then we have $\operatorname{Int}(g)=\operatorname{Int}(\tilde{g})$ and $z^{-n} \in F^{\times}$. Let $\mathbf{z}$ be the element in $\mathrm{H}^{1}\left(F, Z\left(\mathbf{G}_{\mathrm{SC}}\right)\right)$ induced by

$$
\tau(g)^{-1} g=\tau(z)^{-1} z \cdot \mathbf{1}_{n}
$$

for $\tau \in \Gamma$. We remark that the 1-cocycle of $\Gamma$ as in [13, p. 33] is given by $\tau \mapsto g^{-1} \tau(g)$, which is the inverse of ours. We can define $\left\langle\mathbf{z}, s_{\mathbf{m}}\right\rangle$ by using the natural homomorphism $\mathrm{H}^{1}\left(F, Z\left(\mathbf{G}_{\mathrm{SC}}\right)\right) \rightarrow \mathrm{H}^{1}\left(F, \mathbf{T}_{\mathrm{sc}}\right)$. Since $\delta\left(z^{n}\right)$ is equal to the image of $\mathbf{z}$ in $\mathrm{H}^{1}\left(F, \mathbf{T}_{\mathrm{sc}}\right),(3.1)$ implies that

$$
\left\langle\mathbf{z}, s_{\mathbf{m}}\right\rangle=\omega\left(z^{n}\right)=\omega(\operatorname{det} \tilde{g})^{-1} .
$$

Hence, the lemma follows from [13, Section 5.3] and (3.2).

Lemma 3.2. The constant c given by (1.2) does not depend on $\left(\mathbf{B}_{0}, \chi\right)$.

Proof. Let $\left(\mathbf{B}_{0}^{\prime}, \chi^{\prime}\right)$ be another Whittaker datum for $\mathbf{G}$ associated to $\mathbf{s p l} \mathbf{l}_{G}^{\prime}$ and $\psi^{\prime}$, where $\mathbf{s p l}_{G}^{\prime}$ is an $F$-splitting of $\mathbf{G}$ and $\psi^{\prime}$ is a nontrivial character of $F$. By [13, Section 5.3], $\Delta^{\prime}$ does not depend on the choice of $\mathbf{s p l} \mathbf{l}_{G}^{\prime}$ and $\psi^{\prime}$ giving rise to the same $\left(\mathbf{B}_{0}^{\prime}, \chi^{\prime}\right)$. By replacing $\mathbf{s p l}_{G}^{\prime}$ and $\psi^{\prime}$ if necessary, we may assume that $\psi^{\prime}=\psi$. Moreover, by replacing $\mathbf{s p l}_{G}^{\prime}$ by its $G_{\mathrm{SC}}$-conjugate if necessary, we may assume that $\mathbf{s p l}_{G}^{\prime}=\operatorname{Int}(\tilde{t})\left(\mathbf{s p l}_{G}\right)$ for some $\tilde{t} \in T_{0}$. By Lemma 3.1, we have

$$
\operatorname{Tran}_{H}^{\prime}\left(J\left(\pi_{H}\right)\right)=\omega(\operatorname{det} \tilde{t}) \cdot \operatorname{Tran}_{H}^{G}\left(J\left(\pi_{H}\right)\right)
$$

for an irreducible tempered admissible representation $\pi_{H}$ of $H$, where $\operatorname{Tran}_{H}^{\prime} G$ is the transfer with respect to $\left(\mathbf{B}_{0}^{\prime}, \chi^{\prime}\right)$. 
On the other hand, let $\pi$ be the irreducible tempered admissible representation of $G$ such that $\pi \otimes \omega \cong \pi$. Let $\lambda: V_{\pi} \rightarrow \mathbb{C}$ be a Whittaker functional with respect to $\chi$. Since $\chi^{\prime}(u)=\chi\left(\operatorname{Int}\left(\tilde{t}^{-1}\right) u\right)$ for $u \in U_{0}$, the map $\lambda^{\prime}: V_{\pi} \rightarrow \mathbb{C}$ given by $\lambda^{\prime}=\lambda \circ \pi\left(\tilde{t}^{-1}\right)$ is a Whittaker functional with respect to $\chi^{\prime}$. Thus, we obtain

$$
\mathcal{A}_{\omega}^{\prime}=\omega(\operatorname{det} \tilde{t}) \cdot \mathcal{A}_{\omega}
$$

where $\mathcal{A}_{\omega}^{\prime}: V_{\pi} \rightarrow V_{\pi}$ is the intertwining operator with respect to $\lambda^{\prime}$. This yields the lemma.

\section{§4. Reduction to Levi subgroups}

Let $\mathbf{G}=\mathrm{GL}_{n}$, and let $\mathbf{a} \in \mathrm{H}^{1}\left(W_{F}, Z(\hat{G})\right)$. Let $\psi$ be a nontrivial character of $F$. Let $\left(\mathbf{H},{ }^{L} H, s, \xi\right)$ be an endoscopic datum for $(\mathbf{G}, 1, \mathbf{a})$. We fix an $F$-splitting $\mathbf{s p l}_{G}=\left(\mathbf{B}_{0}, \mathbf{T}_{0},\left\{X_{\alpha}\right\}\right)$ (resp., spl $\left.\mathbf{s}_{H}=\left(\mathbf{B}_{H, 0}, \mathbf{T}_{H, 0},\left\{Y_{\alpha}\right\}\right)\right)$ of $\mathbf{G}$ (resp., $\mathbf{H})$ and a $\Gamma$-splitting $\operatorname{spl}_{\hat{G}}=\left(\mathcal{B}, \mathcal{T},\left\{\mathcal{X}_{\check{\alpha}}\right\}\right)$ (resp., spl $\mathbf{l}_{\hat{H}}=\left(\mathcal{B}_{H}, \mathcal{T}_{H}\right.$, $\left.\left\{\mathcal{Y}_{\check{\alpha}}\right\}\right)$ ) of $\hat{G}$ (resp., $\hat{H}$ ). We may assume that the image of $W_{F}$ in $\hat{G}$ under $\xi$ is bounded. Moreover, we may assume that $\xi\left(\mathcal{B}_{H}\right) \subset \mathcal{B}$ and that $\xi\left(\mathcal{T}_{H}\right)=\mathcal{T}$.

We first prove descent to Levi subgroups of $\mathbf{H}$. Let $\mathbf{L}$ be a Levi subgroup of $\mathbf{H}$, and let ${ }^{L} L$ be the Levi subgroup of ${ }^{L} H$ associated to $\mathbf{L}$. Let $\left(\mathbf{L},{ }^{L} L, s_{L}, \xi_{L}\right)$ be the endoscopic datum for $(\mathbf{G}, 1, \mathbf{a})$, where $s_{L}=s \cdot z$ with some $z \in \xi\left(Z(\hat{L})^{0}\right)$ such that the connected centralizer of $s_{L}$ in $\hat{G}$ is $\xi(\hat{L})$, and $\xi_{L}=\left.\xi\right|_{L}$. We take the $F$-splitting $\operatorname{spl}_{L}$ of $\mathbf{L}$ and the $\Gamma$ splitting spl $\mathbf{s}_{\hat{L}}$ of $\hat{L}$ given by $\mathbf{s p l}_{L}=\left(\mathbf{B}_{H, 0} \cap \mathbf{L}, \mathbf{T}_{H, 0},\left\{Y_{\alpha}\right\}_{\alpha \in S(\mathbf{L})}\right)$ and $\mathbf{s p l} \hat{L}_{\hat{L}}=$ $\left(\mathcal{B}_{H} \cap \hat{L}, \mathcal{T}_{H},\left\{\mathcal{Y}_{\check{\alpha}}\right\}_{\alpha \in S(\mathbf{L})}\right)$, respectively. Here $S(\mathbf{L})$ is the set of simple roots of $\mathbf{T}_{H, 0}$ in $\mathbf{B}_{H, 0} \cap \mathbf{L}$. Let $\Delta_{G, H}$ and $\Delta_{G, L}$ be the transfer factors with respect to $\mathbf{s p l}_{G}$ and $\psi$ as in Section 1 .

Lemma 4.1. Let $\gamma \in G$ be a regular semisimple element. Let $\gamma_{H} \in L$ be a $G$-regular semisimple element. If $\gamma_{H}$ is the image of $\gamma$ with respect to the endoscopic datum $\left(\mathbf{L},{ }^{L} L, s_{L}, \xi_{L}\right)$ for $(\mathbf{G}, 1, \mathbf{a})$, then we have

$$
\Delta_{G, L}\left(\gamma_{H}, \gamma\right)=\Delta_{G, H}\left(\gamma_{H}, \gamma\right) \cdot \frac{D_{H}\left(\gamma_{H}\right)}{D_{L}\left(\gamma_{H}\right)}
$$

Proof. Assume that $\gamma_{H}$ is the image of $\gamma$ with respect to $\mathbf{L}$. Let $\mathbf{T}$ (resp., $\mathbf{T}_{H}$ ) be the centralizer of $\gamma$ (resp., $\left.\gamma_{H}\right)$ in $\mathbf{G}$ (resp., $\left.\mathbf{L}\right)$. Let $i_{0}$ : 
$\mathbf{T}_{H, 0} \rightarrow \mathbf{T}_{0}$ be the dual of $\xi^{-1}: \mathcal{T} \rightarrow \mathcal{T}_{H}$. We choose an admissible embedding

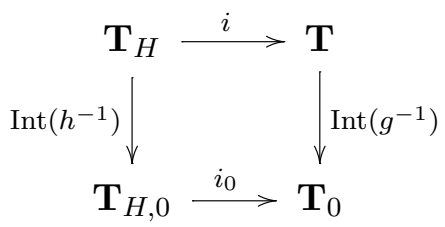

with respect to $\mathbf{L}$ such that $i\left(\gamma_{H}\right)=\gamma$, where $h \in \mathbf{L}_{\mathrm{SC}}(\bar{F})$ and $g \in \mathbf{G}_{\mathrm{SC}}(\bar{F})$. We identify $h$ with its image in $\mathbf{H}_{\mathrm{SC}}(\bar{F})$ and regard $i$ as an admissible embedding with respect to $\mathbf{H}$. We can take $a$-data $\left\{a_{\alpha}\right\}$ and $\chi$-data $\left\{\chi_{\alpha}\right\}$ so that $a_{\alpha}=1$ and $\chi_{\alpha}=\mathbb{1}$ for $\alpha \in R\left(\mathbf{H}, \mathbf{T}_{H}\right)-R\left(\mathbf{L}, \mathbf{T}_{H}\right)$ since such an $\alpha$ is asymmetric (see [15, Section 2.1]).

By definition, we have $\Delta_{G, L, \mathrm{I}}\left(\gamma_{H}, \gamma\right)=\Delta_{G, H, \mathrm{I}}\left(\gamma_{H}, \gamma\right)$ and $\Delta_{G, L, \mathrm{III}_{1}}\left(\gamma_{H}, \gamma\right)$ $=\Delta_{G, H, \mathrm{III}_{1}}\left(\gamma_{H}, \gamma\right)=1$. Since $\chi_{\alpha}=\mathbb{1}$ for $\alpha \in R\left(\mathbf{H}, \mathbf{T}_{H}\right)-R\left(\mathbf{L}, \mathbf{T}_{H}\right)$, we have $\Delta_{G, L, \mathrm{II}}\left(\gamma_{H}, \gamma\right)=\Delta_{G, H, \mathrm{II}}\left(\gamma_{H}, \gamma\right)$.

Let $\bullet=\mathbf{L}$ or $\mathbf{H}$. Let $\xi_{T}^{\bullet}$ and $\xi_{T_{H}}^{\bullet}$ be as in [15, Section 2.6] with respect to •. By definition, we have $\xi_{T}^{L}=\xi_{T}^{H}$. Let $n\left(\omega_{T_{H}}^{\bullet}(\sigma)\right)$ be as in [15, Section 2.6] with respect to $\bullet$. Then we have $\omega_{T_{H}}^{\bullet}(\sigma) \in \Omega\left(\mathbf{L}, \mathbf{T}_{H, 0}\right)=\Omega\left(\hat{L}, \mathcal{T}_{H}\right)$, so that $n\left(\omega_{T_{H}}^{L}(\sigma)\right)=n\left(\omega_{T_{H}}^{H}(\sigma)\right)$ by the definition of $\mathbf{s p l}_{\hat{L}}$. Let $r_{p_{0}}^{\bullet}$ and $s_{p / p_{0}}^{\bullet}$ be as in [15, Section 2.6] with respect to $\bullet$, where $p_{0}$ and $p$ are gauges as in [15, p. 235] and [15, p. 238], respectively. To be precise, we identify $R\left(\bullet, \mathbf{T}_{H}\right)$ with $R\left(\bullet, \mathbf{T}_{H, 0}\right)=\check{R}\left(\hat{\bullet}, \mathcal{T}_{H}\right)$, where $\check{R}\left(\hat{\bullet}, \mathcal{T}_{H}\right)$ is the set of coroots of $\mathcal{T}_{H}$ in $\hat{\bullet}$, and we take gauges $p_{0}^{\bullet}$ on $R\left(\bullet, \mathbf{T}_{H}\right)$ so that the restriction of $p_{0}^{H}$ to $R\left(\mathbf{L}, \mathbf{T}_{H}\right)$ is equal to $p_{0}^{L}$. If $\alpha \in R\left(\mathbf{H}, \mathbf{T}_{H}\right)-R\left(\mathbf{L}, \mathbf{T}_{H}\right)$, then $\alpha$ does not contribute to $r_{p_{0}}^{H}$ since $\chi_{\alpha}=\mathbb{1}$, and $\alpha$ does not contribute to $s_{p / p_{0}}^{H}$ since $\alpha$ is asymmetric. Indeed, we have $p_{0}\left(\tau^{-1}(\alpha)\right)=p_{0}(\alpha)$ and $p\left(\tau^{-1}(\alpha)\right)=p(\alpha)$ for $\tau \in \Gamma$ and $\alpha \in R\left(\mathbf{H}, \mathbf{T}_{H}\right)-R\left(\mathbf{L}, \mathbf{T}_{H}\right)$. Hence, we have $r_{p_{0}}^{L}=r_{p_{0}}^{H}$ and $s_{p / p_{0}}^{L}=s_{p / p_{0}}^{H}$. Thus, we obtain $\xi_{T_{H}}^{L}=\xi_{T_{H}}^{H}$, and hence $\Delta_{G, L, \mathrm{III}_{2}}\left(\gamma_{H}, \gamma\right)=\Delta_{G, H, \mathrm{III}_{2}}\left(\gamma_{H}, \gamma\right)$. This completes the proof.

Let $\phi_{L}$ be a tempered Langlands parameter for $\mathbf{L}$. Let $\phi_{H}$ be the composition of $\phi_{L}$ with the embedding ${ }^{L} L \subset{ }^{L} H$. Put $\phi_{G}=\xi_{L} \circ \phi_{L}$. Let $\pi_{L}$, $\pi_{H}, \pi_{G}$ be the irreducible tempered admissible representations of $L, H, G$ associated to $\phi_{L}, \phi_{H}, \phi_{G}$, respectively. As in (1.2), we define $c_{L}$ and $c_{H}$ by $\operatorname{Tran}_{L}^{G}\left(J\left(\pi_{L}\right)\right)=c_{L} \cdot J^{\omega}\left(\pi_{G}\right)$ and $\operatorname{Tran}_{H}^{G}\left(J\left(\pi_{H}\right)\right)=c_{H} \cdot J^{\omega}\left(\pi_{G}\right)$, respectively.

Lemma 4.2. We have

$$
c_{L}=c_{H}
$$


Proof. By [11, Lemma 8.8] and Lemma 4.1, we have $\operatorname{Tran}_{L}^{G}\left(J\left(\pi_{L}\right)\right)=$ $\operatorname{Tran}_{H}^{G}\left(J\left(\pi_{H}\right)\right)$. This completes the proof.

We next prove descent to Levi subgroups of $\mathbf{G}$. Let $\mathbf{M}$ be a Levi subgroup of $\mathbf{G}$, and let ${ }^{L} M$ be the Levi subgroup of ${ }^{L} G$ associated to $\mathbf{M}$. Assume that $\xi\left({ }^{L} H\right) \subset{ }^{L} M$. We may regard $\left(\mathbf{H},{ }^{L} H, s, \xi\right)$ as an endoscopic datum for $(\mathbf{M}, 1, \mathbf{a})$. We take the $F$-splitting $\mathbf{s p l}_{M}$ of $\mathbf{M}$ and the $\Gamma$-splitting $\mathbf{s p l}_{\hat{M}}$ of $\hat{M}$ given by $\mathbf{s p l}_{M}=\left(\mathbf{B}_{0} \cap \mathbf{M}, \mathbf{T}_{0},\left\{X_{\alpha}\right\}_{\alpha \in S(\mathbf{M})}\right)$ and $\mathbf{s p l}_{\hat{M}}=(\mathcal{B} \cap$ $\hat{M}, \mathcal{T},\left\{\mathcal{X}_{\check{\alpha}}\right\}_{\alpha \in S(\mathbf{M})}$, respectively. Here $S(\mathbf{M})$ is the set of simple roots of $\mathbf{T}_{0}$ in $\mathbf{B}_{0} \cap \mathbf{M}$. Let $\Delta_{G, H}$ (resp., $\Delta_{M, H}$ ) be the transfer factor with respect to $\mathbf{s p l}_{G}$ and $\psi$ (resp., $\mathbf{s p l}_{M}$ and $\psi$ ) as in Section 1 .

LEMmA 4.3. Let $\gamma \in M$ be a $G$-regular semisimple element. Let $\gamma_{H} \in H$ be a G-regular semisimple element. If $\gamma_{H}$ is the image of $\gamma$ with respect to the endoscopic datum $\left(\mathbf{H},{ }^{L} H, s, \xi\right)$ for $(\mathbf{M}, 1, \mathbf{a})$, then we have

$$
\Delta_{M, H}\left(\gamma_{H}, \gamma\right)=\Delta_{G, H}\left(\gamma_{H}, \gamma\right) \cdot \frac{D_{M}(\gamma)}{D_{G}(\gamma)}
$$

Proof. Assume that $\gamma_{H}$ is the image of $\gamma$ with respect to $\mathbf{M}$. Let $\mathbf{T}$ (resp., $\mathbf{T}_{H}$ ) be the centralizer of $\gamma\left(\right.$ resp., $\left.\gamma_{H}\right)$ in $\mathbf{M}$ (resp., $\left.\mathbf{H}\right)$. Let $i_{0}$ : $\mathbf{T}_{H, 0} \rightarrow \mathbf{T}_{0}$ be the dual of $\xi^{-1}: \mathcal{T} \rightarrow \mathcal{T}_{H}$. We choose an admissible embedding

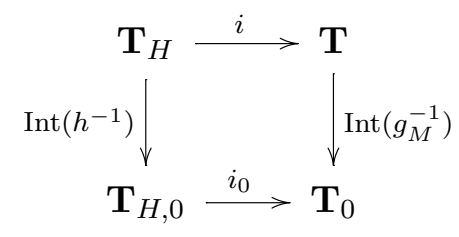

with respect to $\mathbf{M}$ such that $i\left(\gamma_{H}\right)=\gamma$, where $h \in \mathbf{H}_{\mathrm{SC}}(\bar{F})$ and $g_{M} \in$ $\mathbf{M}_{\mathrm{SC}}(\bar{F})$. We regard $i$ as an admissible embedding with respect to $\mathbf{G}$ by using the image $g_{G}$ of $g_{M}$ in $\mathbf{G}_{\mathrm{SC}}(\bar{F})$. We can take $a$-data $\left\{a_{\alpha}\right\}$ and $\chi$-data $\left\{\chi_{\alpha}\right\}$ so that $a_{\alpha}=1$ and $\chi_{\alpha}=\mathbb{1}$ for $\alpha \in R(\mathbf{G}, \mathbf{T})-R(\mathbf{M}, \mathbf{T})$ since such an $\alpha$ is asymmetric (see [15, Section 2.1]).

By definition, we have $\Delta_{M, H, \mathrm{III}_{1}}\left(\gamma_{H}, \gamma\right)=\Delta_{G, H, \mathrm{III}_{1}}\left(\gamma_{H}, \gamma\right)=1$. Since $\chi_{\alpha}=\mathbb{1}$ for $\alpha \in R(\mathbf{G}, \mathbf{T})-R(\mathbf{M}, \mathbf{T})$, we have $\Delta_{M, H, \mathrm{II}}\left(\gamma_{H}, \gamma\right)=\Delta_{G, H, \mathrm{II}}\left(\gamma_{H}, \gamma\right)$.

Let $\bullet=\mathbf{M}$ or $\mathbf{G}$. Let $\mathbf{T}_{\mathrm{sc}}^{\bullet}\left(\operatorname{resp} ., \mathbf{T}_{0, \mathrm{sc}}^{\bullet}\right)$ be the preimage of $\mathbf{T}\left(\operatorname{resp} ., \mathbf{T}_{0}\right)$ in •SC. Let $m\left(\sigma_{T_{\mathrm{sc}}^{\bullet}}\right)=x\left(\sigma_{T_{\mathrm{sc}}^{\bullet}}\right) n\left(\omega_{T_{\mathrm{sc}}}(\sigma)\right)$ and $\lambda\left(\mathbf{T}_{\mathrm{SC}}^{\bullet}\right) \in \mathrm{H}^{1}\left(F, \mathbf{T}_{\mathrm{sc}}^{\bullet}\right)$ be as in $[15$, Section 2.3]. Then the image of $\omega_{T_{\mathrm{sc}}^{M}}(\sigma)=\operatorname{Int}\left(g_{M}^{-1} \sigma\left(g_{M}\right)\right) \in \Omega\left(\mathbf{M}_{\mathrm{SC}}, \mathbf{T}_{0, \mathrm{sc}}^{M}\right)$ 
in $\Omega\left(\mathbf{G}_{\mathrm{SC}}, \mathbf{T}_{0, \mathrm{sc}}^{G}\right)$ is equal to $\omega_{T_{\mathrm{sc}}^{G}}(\sigma)=\operatorname{Int}\left(g_{G}^{-1} \sigma\left(g_{G}\right)\right)$, so that the image of $n\left(\omega_{T_{\mathrm{sc}}^{M}}(\sigma)\right)$ in $\mathbf{G}_{\mathrm{SC}}(\bar{F})$ is equal to $n\left(\omega_{T_{\mathrm{sc}}^{G}}(\sigma)\right)$ by the definition of $\mathbf{s p l}_{M}$. Since $a_{\alpha}=1$ for $\alpha \in R(\mathbf{G}, \mathbf{T})-R(\mathbf{M}, \mathbf{T})$, the image of $x\left(\sigma_{T_{\mathrm{sc}}^{M}}\right)$ in $\mathbf{T}_{\mathrm{sc}}^{G}(\bar{F})$ is equal to $x\left(\sigma_{T_{\mathrm{sc}}^{G}}\right)$. Hence, the image of $m\left(\sigma_{T_{\mathrm{sc}}^{M}}\right)$ in $\mathbf{G}_{\mathrm{SC}}(\bar{F})$ is equal to $m\left(\sigma_{T_{\mathrm{sc}}^{G}}\right)$, so that the image of $\lambda\left(\mathbf{T}_{\mathrm{sc}}^{M}\right)$ in $\mathrm{H}^{1}\left(F, \mathbf{T}_{\mathrm{sc}}^{G}\right)$ is equal to $\lambda\left(\mathbf{T}_{\mathrm{sc}}^{G}\right)$. Let $\mathbf{s}_{T}^{\bullet} \in \pi_{0}\left((\hat{T} / Z(\hat{\bullet}))^{\Gamma}\right)$ be the image of $s \in \mathcal{T}$ under

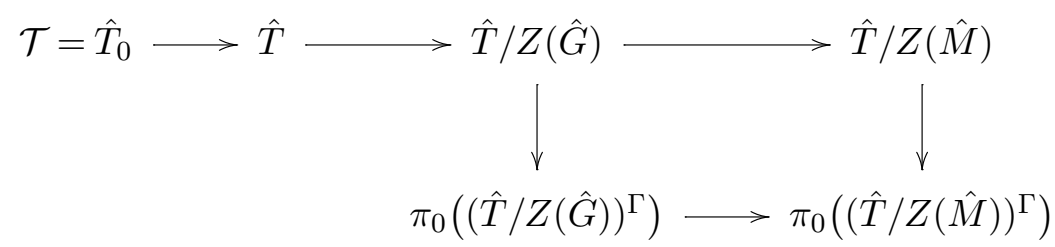

In particular, the image of $\mathbf{s}_{T}^{G}$ in $\pi_{0}\left((\hat{T} / Z(\hat{M}))^{\Gamma}\right)$ is equal to $\mathbf{s}_{T}^{M}$. Thus, we obtain

$$
\Delta_{M, H, \mathrm{I}}\left(\gamma_{H}, \gamma\right)=\left\langle\lambda\left(T_{\mathrm{sc}}^{M}\right), \mathbf{s}_{T}^{M}\right\rangle=\left\langle\lambda\left(T_{\mathrm{sc}}^{G}\right), \mathbf{s}_{T}^{G}\right\rangle=\Delta_{G, H, \mathrm{I}}\left(\gamma_{H}, \gamma\right)
$$

Let $\xi_{T}^{\bullet}$ and $\xi_{T_{H}}$ be as in [15, Section 2.6] with respect to $\bullet$. By definition, we have $\xi_{T_{H}}^{M}=\xi_{T_{H}}^{G}$. Let $n\left(\omega_{T}^{\bullet}(\sigma)\right)$ be as in [15, Section 2.6] with respect to $\bullet$. Then the image of $\omega_{T}^{M}(\sigma) \in \Omega\left(\mathbf{M}, \mathbf{T}_{0}\right)=\Omega(\hat{M}, \mathcal{T})$ in $\Omega(\hat{G}, \mathcal{T})$ is equal to $\omega_{T}^{G}(\sigma)$, so that $n\left(\omega_{T}^{M}(\sigma)\right)=n\left(\omega_{T}^{G}(\sigma)\right)$ by the definition of $\mathbf{s p l} \mathbf{l}_{\hat{M}}$. Let $r_{p_{0}}^{\bullet}$ and $s_{p / p_{0}}^{\bullet}$ be as in $[15$, Section 2.6] with respect to $\bullet$. To be precise, we take gauges $p_{0}^{\bullet}$ on $R(\bullet, \mathbf{T})$ so that the restriction of $p_{0}^{G}$ to $R(\mathbf{M}, \mathbf{T})$ is equal to $p_{0}^{M}$. If $\alpha \in R(\mathbf{G}, \mathbf{T})-R(\mathbf{M}, \mathbf{T})$, then $\alpha$ does not contribute to $r_{p_{0}}^{G}$ since $\chi_{\alpha}=\mathbb{1}$, and $\alpha$ does not contribute to $s_{p / p_{0}}^{G}$ since $\alpha$ is asymmetric. Hence, we have $r_{p_{0}}^{M}=r_{p_{0}}^{G}$ and $s_{p / p_{0}}^{M}=s_{p / p_{0}}^{G}$. Thus, we obtain $\xi_{T}^{M}=\xi_{T}^{G}$ and $\Delta_{M, H, \mathrm{III}_{2}}\left(\gamma_{H}, \gamma\right)=\Delta_{G, H, \mathrm{III}_{2}}\left(\gamma_{H}, \gamma\right)$. This completes the proof.

Let $\phi_{H}$ be a tempered Langlands parameter for $\mathbf{H}$. Put $\phi_{G}=\xi \circ \phi_{H}$. We may regard $\phi_{G}$ as a tempered Langlands parameter $\phi_{M}$ for $\mathbf{M}$. Let $\pi_{H}$, $\pi_{M}, \pi_{G}$ be the irreducible tempered admissible representations of $H, M, G$ associated to $\phi_{H}, \phi_{M}, \phi_{G}$, respectively.

Let $\mathbf{U}_{0}$ be the unipotent radical of $\mathbf{B}_{0}$, and let $\chi$ be the generic character associated to $\operatorname{spl}_{G}$ and $\psi$. Put $\chi^{M}=\left.\chi\right|_{U_{0} \cap M}$. Then $\chi^{M}$ is the generic character associated to $\mathbf{s p l}_{M}$ and $\psi$. Let $\mathcal{A}_{\omega}^{M}: V_{\pi_{M}} \rightarrow V_{\pi_{M}}$ be the intertwining 
operator such that $\mathcal{A}_{\omega}^{M} \circ\left(\pi_{M} \otimes \omega\right)=\pi_{M} \circ \mathcal{A}_{\omega}^{M}$ and $\lambda^{M} \circ \mathcal{A}_{\omega}^{M}=\lambda^{M}$, where $\lambda^{M}: V_{\pi_{M}} \rightarrow \mathbb{C}$ is a Whittaker functional with respect to $\chi^{M}$.

We now construct the intertwining operator $\mathcal{A}_{\omega}^{G}: V_{\pi_{G}} \rightarrow V_{\pi_{G}}$ explicitly. We may assume that

$$
\mathbf{M}=\left\{\operatorname{diag}\left(g_{1}, \ldots, g_{r}\right) \mid g_{1} \in \mathrm{GL}_{m_{1}}, \ldots, g_{r} \in \mathrm{GL}_{m_{r}}\right\}
$$

where $m_{1}+\cdots+m_{r}=n$. We can take the standard splittings $\mathbf{s p l}_{G}$ and $\mathbf{s p l} \mathbf{p l}_{M}$ of $\mathbf{G}$ and $\mathbf{M}$, respectively. Then we have $\pi_{G}=\operatorname{Ind}_{P}^{G}\left(\pi_{M}\right)$, where $\mathbf{P}=\mathbf{M N}$ is the standard parabolic subgroup of $\mathbf{G}$ and the space $V_{\pi_{G}}$ consists of locally constant $V_{\pi_{M}}$-valued functions $\Phi$ on $G$ such that

$$
\Phi(m n g)=\delta_{P}^{1 / 2}(m) \pi_{M}(m) \Phi(g)
$$

for $m \in M, n \in N, g \in G$. Here $\delta_{P}$ is the modulus character of $P$. We define $\mathcal{A}_{\omega}^{G}$ by

$$
\left(\mathcal{A}_{\omega}^{G} \Phi\right)(g)=\omega(g) \mathcal{A}_{\omega}^{M} \Phi(g)
$$

for $\Phi \in V_{\pi_{G}}$ and $g \in G$. Then we have $\mathcal{A}_{\omega}^{G} \circ\left(\pi_{G} \otimes \omega\right)=\pi_{G} \circ \mathcal{A}_{\omega}^{G}$.

LEMMA 4.4. We have

$$
\lambda^{G} \circ \mathcal{A}_{\omega}^{G}=\lambda^{G}
$$

where $\lambda^{G}: V_{\pi_{G}} \rightarrow \mathbb{C}$ is a Whittaker functional with respect to $\chi$.

Proof. By [16, Proposition 3.1], we may assume that the Whittaker functional $\lambda^{G}$ is given by

$$
\lambda^{G}(\Phi)=\int_{U^{\prime}} \lambda^{M}\left(\Phi\left(w_{0} u^{\prime}\right)\right) \overline{\chi\left(u^{\prime}\right)} d u^{\prime}
$$

for $\Phi \in V_{\pi_{G}}$, where

$$
w_{0}=\left(\begin{array}{ll} 
& \mathbf{1}_{m_{1}} \\
\mathbf{1}_{m_{r}} &
\end{array}\right)
$$

and

$$
\mathbf{U}^{\prime}=\left\{\left(\begin{array}{ccc}
\mathbf{1}_{m_{r}} & * & * \\
& \ddots & * \\
& & \mathbf{1}_{m_{1}}
\end{array}\right)\right\}
$$


Since $\pi_{M} \otimes \omega \cong \pi_{M}$, the order of $\omega$ divides $m_{1}, \ldots, m_{r}$, so that $\omega\left(w_{0}\right)=1$. Hence, we have

$$
\begin{aligned}
\lambda^{G}\left(\mathcal{A}_{\omega}^{G} \Phi\right) & =\int_{U^{\prime}} \lambda^{M}\left(\mathcal{A}_{\omega}^{G} \Phi\left(w_{0} u^{\prime}\right)\right) \overline{\chi\left(u^{\prime}\right)} d u^{\prime} \\
& =\int_{U^{\prime}} \lambda^{M}\left(\mathcal{A}_{\omega}^{M} \omega\left(w_{0} u^{\prime}\right) \Phi\left(w_{0} u^{\prime}\right)\right) \overline{\chi\left(u^{\prime}\right)} d u^{\prime} \\
& =\int_{U^{\prime}} \lambda^{M}\left(\mathcal{A}_{\omega}^{M} \Phi\left(w_{0} u^{\prime}\right)\right) \overline{\chi\left(u^{\prime}\right)} d u^{\prime} \\
& =\int_{U^{\prime}} \lambda^{M}\left(\Phi\left(w_{0} u^{\prime}\right)\right) \overline{\chi\left(u^{\prime}\right)} d u^{\prime} \\
& =\lambda^{G}(\Phi) .
\end{aligned}
$$

As in (1.2), we define $c_{M}$ and $c_{G}$ by $\operatorname{Tran}_{H}^{M}\left(J\left(\pi_{H}\right)\right)=c_{M} \cdot J^{\omega}\left(\pi_{M}\right)$ and $\operatorname{Tran}_{H}^{G}\left(J\left(\pi_{H}\right)\right)=c_{G} \cdot J^{\omega}\left(\pi_{G}\right)$, respectively.

LEMma 4.5. We have

$$
c_{M}=c_{G} .
$$

Proof. By [11, Lemma 8.6] and Lemmas 4.3 and 4.4, $\operatorname{Tran}_{H}^{M}\left(J\left(\pi_{H}\right)\right)=$ $c_{M} \cdot J^{\omega}\left(\pi_{M}\right)$ implies that

$$
\operatorname{Tran}_{H}^{G}\left(J\left(\pi_{H}\right)\right)=c_{M} \cdot J^{\omega}\left(\pi_{G}\right)
$$

This completes the proof.

\section{§5. Reduction to tamely ramified cyclic extensions}

Let $E$ be a cyclic extension of $F$ of degree $d$. We first refine a result of Henniart [4, lemme 3.6], [5, Section 3].

LEMMA 5.1. There exist a cyclic extension $\mathbb{E}$ of a number field $\mathbb{F}$ of degree $d$ and a place $v_{0}$ of $\mathbb{F}$ such that

- $v_{0}$ is inert in $\mathbb{E}$, and $\mathbb{E}_{v_{0}} / \mathbb{F}_{v_{0}}$ is isomorphic to $E / F$;

- $\mathbb{E}_{w} / \mathbb{F}_{v}$ is tamely ramified if $v \neq v_{0}$ is a nonarchimedean place of $\mathbb{F}$ and $w$ is a place of $\mathbb{E}$ lying above $v$; and

- $\mathbb{F}_{v} \cong \mathbb{C}$ if $v$ is an archimedean place of $\mathbb{F}$.

Proof. We first recall the Grunwald-Wang theorem, from which the lemma follows. Let $\mathbb{F}$ be a number field. For each positive integer $r$, fix a primitive $2^{r}$ th root of unity $\xi_{r}$ such that $\xi_{r+1}^{2}=\xi_{r}$. Put $\eta_{r}=\xi_{r}+\xi_{r}^{-1}$. Note that $\eta_{2}=0$. 
Let $s \geq 2$ be the integer such that $\eta_{s} \in \mathbb{F}$ but $\eta_{s+1} \notin \mathbb{F}$. Let $S_{0}$ be the set of places $v$ of $\mathbb{F}$ lying above 2 such that $-1,2+\eta_{s},-\left(2+\eta_{s}\right)$ are not square in $\mathbb{F}_{v}$. Let $S$ be a finite set of places of $\mathbb{F}$, and let $d$ be a positive integer. We say that we are in the special case if

- $-1,2+\eta_{s},-\left(2+\eta_{s}\right)$ are not square in $\mathbb{F}$,

- $2^{s+1} \mid d$,

- $S_{0} \subset S$.

For $v \in S$, let $\omega_{v}$ be a character of $\mathbb{F}_{v}^{\times}$of order dividing $d$. Assume that

$$
\prod_{v \in S_{0}} \omega_{v}\left(2+\eta_{s}\right)^{d / 2}=1
$$

if we are in the special case. Then, by the Grunwald-Wang theorem, there exists a character $\boldsymbol{\omega}$ of $\mathbb{A}_{\mathbb{F}}^{\times} / \mathbb{F}^{\times}$of order dividing $d$ such that $\boldsymbol{\omega}_{v}=\omega_{v}$ for $v \in S$ (see [2, Chapter X, Section 2, Theorem 5]).

Let $p$ be the residual characteristic of $F$. Fix a character $\omega$ of $F^{\times}$of order $d$ associated to $E / F$ by class field theory.

We first assume that $p \neq 2$. By the weak approximation theorem and Krasner's lemma, we can take a number field $\mathbb{F}$ and a place $v_{0}$ of $\mathbb{F}$ so that $\mathbb{F}_{v_{0}} \cong F$ and $\mathbb{F}_{v} \cong \mathbb{C}$ if $v$ is an archimedean place of $\mathbb{F}$. Let $S_{\infty}$ be the set of archimedean places of $\mathbb{F}$, and let $S^{\prime}$ the set of nonarchimedean places $v$ of $\mathbb{F}$ such that the residual characteristic of $\mathbb{F}_{v}$ divides $2 d$. Put $S=S_{\infty} \cup S^{\prime} \cup\left\{v_{0}\right\}$. Then we have $S_{0} \subset S$ and $v_{0} \notin S_{0}$. We take

$$
\omega_{v}= \begin{cases}\omega & \text { if } v=v_{0}, \\ \mathbb{1} & \text { if } v \neq v_{0} .\end{cases}
$$

Then (5.1) holds, and hence there exists a character $\boldsymbol{\omega}$ of $\mathbb{A}_{\mathbb{F}}^{\times} / \mathbb{F}^{\times}$of order $d$ such that $\boldsymbol{\omega}_{v}=\omega_{v}$ for $v \in S$. Let $\mathbb{E}$ be the cyclic extension of $\mathbb{F}$ of degree $d$ associated to $\boldsymbol{\omega}$ by class field theory. By construction, $v_{0}$ is inert in $\mathbb{E}$, $\mathbb{E}_{v_{0}} / \mathbb{F}_{v_{0}}$ is isomorphic to $E / F$, and $\mathbb{E}_{w} \cong \mathbb{F}_{v}$ for $v \in S-\left\{v_{0}\right\}$. Here $w$ is a place of $\mathbb{E}$ lying above $v$. If $v \notin S$, then the residual characteristic of $\mathbb{F}_{v}$ does not divide $d$, so that $\mathbb{E}_{w} / \mathbb{F}_{v}$ is tamely ramified.

We next assume that $p=2$. We modify the argument above as follows.

Let $\breve{F}$ be the unramified extension of $\mathbb{Q}_{2}$ of degree $\left[F: \mathbb{Q}_{2}\right]$. Since $\mathbb{Q}_{2}\left(\xi_{r}\right)$ is a totally ramified extension of $\mathbb{Q}_{2}$ of degree $2^{r-1}$, we have $\eta_{r} \notin \breve{F}$ if $r \geq 3$. Also, $-1,2,-2$ are not square in $\breve{F}$. Recall that 2 is split in $\mathbb{Q}(\sqrt{-7})$. By the weak approximation theorem and Krasner's lemma, we can take an extension $\mathbb{F}$ of $\mathbb{Q}(\sqrt{-7})$ and two places $v_{0}, v_{1}$ of $\mathbb{F}$ so that $\mathbb{F}_{v_{0}} \cong F, \mathbb{F}_{v_{1}} \cong \breve{F}$, 
and $\mathbb{F}_{v} \cong \mathbb{C}$ if $v$ is an archimedean place of $\mathbb{F}$. In particular, $s=2$ and $v_{1} \in S_{0}$. Put $S=S_{\infty} \cup S^{\prime} \cup\left\{v_{0}\right\}$. Then we have $S_{0} \subset S$. Moreover, we are in the special case if and only if $8 \mid d$.

For $v \in S$, we choose a character $\omega_{v}$ of $\mathbb{F}_{v}^{\times}$as follows. Fix an unramified character $\breve{\omega}$ of $\breve{F}^{\times}$of order $d$. Note that $\breve{\omega}(2)^{d / 2}=-1$ if $d$ is even. If we are in the special case, $v_{0} \in S_{0}$, and $\omega(2)^{d / 2}=-1$, we take

$$
\omega_{v}= \begin{cases}\omega & \text { if } v=v_{0}, \\ \breve{\omega} & \text { if } v=v_{1}, \\ \mathbb{1} & \text { if } v \notin\left\{v_{0}, v_{1}\right\} .\end{cases}
$$

Otherwise, we take

$$
\omega_{v}= \begin{cases}\omega & \text { if } v=v_{0} \\ \mathbb{1} & \text { if } v \neq v_{0}\end{cases}
$$

Then (5.1) holds if we are in the special case. Indeed, if we are in the special case and $v_{0} \in S_{0}$, then we have

$$
\prod_{v \in S_{0}} \omega_{v}\left(2+\eta_{s}\right)^{d / 2}=\omega(2)^{d / 2} \cdot \omega_{v_{1}}(2)^{d / 2}=1 .
$$

Hence, there exists a character $\boldsymbol{\omega}$ of $\mathbb{A}_{\mathbb{F}}^{\times} / \mathbb{F}^{\times}$of order $d$ such that $\boldsymbol{\omega}_{v}=\omega_{v}$ for $v \in S$. Let $\mathbb{E}$ be the cyclic extension of $\mathbb{F}$ of degree $d$ associated to $\boldsymbol{\omega}$ by class field theory. By construction, if we are in the special case, $v_{0} \in S_{0}$, and $\omega(2)^{d / 2}=-1$, then $v_{1}$ is inert in $\mathbb{E}$ and $\mathbb{E}_{v_{1}} / \mathbb{F}_{v_{1}}$ is unramified. This completes the proof.

Let $\omega$ be a character of $F^{\times}$of order $d$ associated to $E / F$ by class field theory. Fix a character $\boldsymbol{\omega}$ of $\mathbb{A}_{\mathbb{F}}^{\times} / \mathbb{F}^{\times}$of order $d$ associated to $\mathbb{E} / \mathbb{F}$ by class field theory such that $\boldsymbol{\omega}_{v_{0}}=\omega$. Let $\mathbf{G}=\mathrm{GL}_{d}$, and let $\mathbf{H}=\operatorname{Res}_{\mathbb{E} / \mathbb{F}} \mathbb{G}_{m}$. We fix an $\mathbb{F}$-splitting $\mathbf{s p l}_{G}$ of $\mathbf{G}$ and a nontrivial character $\boldsymbol{\psi}$ of $\mathbb{A}_{\mathbb{F}} / \mathbb{F}$. For each place $v$ of $\mathbb{F}, \mathbf{s p l}_{G}$ and $\boldsymbol{\psi}$ induce an $\mathbb{F}_{v}$-splitting $\mathbf{s p l}_{G}$ of $\mathrm{GL}_{d}$ and a nontrivial character of $\boldsymbol{\psi}_{v}$ of $\mathbb{F}_{v}$, respectively.

We now recall a special case of a result of Henniart and Lemaire [9] on the product formula for the constant.

Proposition 5.2 [9, section 4.6]. Let $\pi_{H}$ be a unitary character of $E^{\times}$. Then there exists a unitary character $\Pi_{H}=\bigotimes_{v} \Pi_{H, v}$ of $\mathbb{A}_{\mathbb{E}}^{\times} / \mathbb{E}^{\times}$such that $\Pi_{H, v_{0}}=\pi_{H}$ and

$$
\prod_{v \in S} c_{v}=1
$$


for a sufficiently large finite set $S$ of places of $\mathbb{F}$ which contains $v_{0}$. Here $c_{v} \in \mathbb{C}^{\times}$is the constant for $\Pi_{H, v}$ given by (1.2).

\section{$\S 6$. Intertwining operators}

To prove Theorem 1.4, we may assume that

- $E$ is a tamely ramified cyclic extension of $F$ of degree $d$,

- $\mathbf{G}=\mathrm{GL}_{d}$

- $(\mathbf{H}, \mathcal{H}, s, \xi)=\left(\operatorname{Res}_{E / F} \mathbb{G}_{m},{ }^{L} H_{\mathbf{m}}, s_{\mathbf{m}}, \xi_{\mathbf{m}}\right)$ with $\mathbf{m}=(1)$,

- $\operatorname{spl}_{G}$ is standard,

- $\psi$ is of order zero, and

- $\pi_{H}=\mathbb{1}$

by Theorem 1.2, Proposition 5.2, and Lemmas 2.1, 2.3, 3.2, 4.2, 4.5, and 5.1. The rest of this paper is devoted to the proof of Theorem 1.4 under this assumption. In this section, we construct the intertwining operator $\mathcal{A}_{\omega}$ explicitly.

Let $e$ and $f$ be the ramification index and the residual degree of $E / F$, respectively. Note that $d=e f$. Fix a character $\omega$ of $F^{\times}$of order $d$ associated to $E / F$ by class field theory. Then $\omega$ is trivial on $1+\mathfrak{p}$ and the restriction of $\omega$ to $\mathfrak{o}^{\times}$is of order $e$.

Recall that $\mathbf{B}_{0}=\mathbf{T}_{0} \mathbf{U}_{0}$ is the standard Borel subgroup of $\mathbf{G}$, where $\mathbf{T}_{0}$ is the subgroup of diagonal matrices and

$$
\mathbf{U}_{0}=\left\{u=\left(u_{i, j}\right) \in \mathbf{G} \mid u_{i, i}=1, u_{i, j}=0 \text { if } i>j\right\} .
$$

The generic character $\chi$ of $U_{0}$ associated to $\operatorname{spl}_{G}$ and $\psi$ is given by

$$
\chi(u)=\psi\left(u_{1,2}+\cdots+u_{d-1, d}\right)
$$

for $u \in U_{0}$.

Let $\mu$ be the character of $T_{0}$ defined by

$$
\begin{aligned}
& \mu=\mathbb{1} \otimes \omega^{e} \otimes \cdots \otimes \omega^{e(f-1)} \otimes \omega \otimes \omega^{e+1} \otimes \cdots \otimes \omega^{e(f-1)+1} \otimes \cdots \otimes \omega^{e-1} \\
& \otimes \omega^{2 e-1} \otimes \cdots \otimes \omega^{e f-1} .
\end{aligned}
$$

For $s=\left(s_{1}, \ldots, s_{d}\right) \in \mathbb{C}^{d}$, we put

$$
\mu_{s}=\mu|\cdot|^{s},
$$

where $|t|^{s}=\left|t_{1}\right|^{s_{1}} \cdots\left|t_{d}\right|^{s_{d}}$ for $t=\operatorname{diag}\left(t_{1}, \ldots, t_{d}\right) \in T_{0}$. Let

$$
\pi_{s}=\operatorname{Ind}_{B_{0}}^{G}\left(\mu_{s}\right)
$$


be the principal series representation of $G$ on the space $V_{s}$ of locally constant functions $\Phi_{s}$ such that

$$
\Phi_{s}(t u g)=\delta_{B_{0}}^{1 / 2}(t) \mu_{s}(t) \Phi_{s}(g)
$$

for $t \in T_{0}, u \in U_{0}, g \in G$. Here $\delta_{B_{0}}$ is the modulus character of $B_{0}$, and $G$ acts on $V_{s}$ by right translation. Let

$$
\lambda_{s}: V_{s} \longrightarrow \mathbb{C}
$$

be the Whittaker functional given by

$$
\lambda_{s}\left(\Phi_{s}\right)=\int_{U_{0}} \Phi_{s}\left(w_{0} u\right) \overline{\chi(u)} d u
$$

where

$$
w_{0}=\left(\begin{array}{cccc}
0 & \cdots & 0 & 1 \\
0 & \cdots & 1 & 0 \\
\vdots & . \cdot & \vdots & \vdots \\
1 & \cdots & 0 & 0
\end{array}\right)
$$

and $d u$ is the Haar measure on $U_{0}$ such that $\operatorname{vol}\left(\mathbf{U}_{0}(\mathfrak{o})\right)=1$. We write

$$
\pi=\pi_{0}, \quad V=V_{0}, \quad \lambda=\lambda_{0} .
$$

Then $\pi$ is the automorphic induction of the trivial representation of $H$.

Let $\mu^{\prime}$ be the character of $T_{0}$ defined by

$$
\begin{aligned}
\mu^{\prime}= & \omega \otimes \omega^{e+1} \otimes \cdots \otimes \omega^{e(f-1)+1} \otimes \cdots \otimes \omega^{e-1} \otimes \omega^{2 e-1} \otimes \cdots \otimes \omega^{e f-1} \otimes \omega^{e} \\
& \otimes \cdots \otimes \omega^{e(f-1)} \otimes \mathbb{1} .
\end{aligned}
$$

Note that $\mu^{\prime}=\mu \omega$. For $s \in \mathbb{C}^{d}$, we put $\mu_{s}^{\prime}=\mu^{\prime}|\cdot|^{s}$. We define a principal series representation $\pi_{s}^{\prime}=\operatorname{Ind}_{B_{0}}^{G}\left(\mu_{s}^{\prime}\right)$ of $G$ on the space $V_{s}^{\prime}$ and a Whittaker functional

$$
\lambda_{s}^{\prime}: V_{s}^{\prime} \longrightarrow \mathbb{C}
$$

similarly. We write

$$
\pi^{\prime}=\pi_{0}^{\prime}, \quad V^{\prime}=V_{0}^{\prime}, \quad \lambda^{\prime}=\lambda_{0}^{\prime} .
$$

We define an isomorphism

$$
\mathcal{A}: V \longrightarrow V^{\prime}
$$


as vector spaces by

$$
\mathcal{A}(\Phi)(g)=\Phi(g) \omega(g)
$$

for $\Phi \in V$ and $g \in G$. Here we write $\omega(g)=\omega(\operatorname{det} g)$ for $g \in G$.

LEMMA 6.1. We have

$$
\mathcal{A} \circ(\pi \otimes \omega)=\pi^{\prime} \circ \mathcal{A}
$$

Proof. Let $\Phi \in V$, and let $g, g^{\prime} \in G$. Then we have

$$
\begin{aligned}
\mathcal{A}\left((\pi \otimes \omega)\left(g^{\prime}\right) \Phi\right)(g) & =\mathcal{A}\left(\pi\left(g^{\prime}\right) \Phi\right)(g) \omega\left(g^{\prime}\right)=\pi\left(g^{\prime}\right) \Phi(g) \omega(g) \omega\left(g^{\prime}\right) \\
& =\Phi\left(g g^{\prime}\right) \omega\left(g g^{\prime}\right)
\end{aligned}
$$

and

$$
\pi^{\prime}\left(g^{\prime}\right) \mathcal{A}(\Phi)(g)=\mathcal{A}(\Phi)\left(g g^{\prime}\right)=\Phi\left(g g^{\prime}\right) \omega\left(g g^{\prime}\right) .
$$

LEMMA 6.2. We have

$$
\lambda^{\prime} \circ \mathcal{A}=\omega(-1)^{d(d-1) / 2} \cdot \lambda
$$

Proof. Let $\Phi \in V$. Then we have

$$
\begin{aligned}
\lambda^{\prime}(\mathcal{A}(\Phi)) & =\int_{U_{0}} \mathcal{A}(\Phi)\left(w_{0} u\right) \overline{\chi(u)} d u \\
& =\int_{U_{0}} \Phi\left(w_{0} u\right) \omega\left(w_{0} u\right) \overline{\chi(u)} d u \\
& =\omega\left(w_{0}\right) \int_{U_{0}} \Phi\left(w_{0} u\right) \overline{\chi(u)} d u \\
& =\omega\left(w_{0}\right) \cdot \lambda(\Phi) .
\end{aligned}
$$

Let $\mathbf{P}$ be the standard parabolic subgroup of $\mathbf{G}$ with Levi factor

$$
\mathbf{L}=\left\{\left(\begin{array}{cc}
g_{1} & 0 \\
0 & g_{2}
\end{array}\right) \mid g_{1} \in \mathrm{GL}_{d-f}, g_{2} \in \mathrm{GL}_{f}\right\}
$$

Let

$$
\begin{aligned}
& \mathbf{L}_{1}=\left\{\operatorname{diag}\left(g_{1}, \mathbf{1}_{f}\right) \in \mathbf{L} \mid g_{1} \in \mathrm{GL}_{d-f}\right\} \\
& \mathbf{L}_{2}=\left\{\operatorname{diag}\left(\mathbf{1}_{d-f}, g_{2}\right) \in \mathbf{L} \mid g_{2} \in \mathrm{GL}_{f}\right\} .
\end{aligned}
$$


For $i=1,2$, put

$$
\pi_{s, i}^{\prime}=\operatorname{Ind}_{B_{0} \cap L_{i}}^{L_{i}}\left(\mu_{s, i}^{\prime}\right)
$$

where $\mu_{s, i}^{\prime}=\left.\mu_{s}^{\prime}\right|_{B_{0} \cap L_{i}}$. We write $\pi_{i}^{\prime}=\pi_{0, i}^{\prime}$ and $\mu_{i}^{\prime}=\mu_{0, i}^{\prime}$. Note that

$$
\pi_{s}^{\prime} \cong \operatorname{Ind}_{P}^{G}\left(\pi_{s, 1}^{\prime} \otimes \pi_{s, 2}^{\prime}\right)
$$

and that

$$
\begin{aligned}
& \mu_{1}^{\prime}=\omega \otimes \omega^{e+1} \otimes \cdots \otimes \omega^{e(f-1)+1} \otimes \cdots \otimes \omega^{e-1} \otimes \omega^{2 e-1} \otimes \cdots \otimes \omega^{e f-1}, \\
& \mu_{2}^{\prime}=\omega^{e} \otimes \cdots \otimes \omega^{e(f-1)} \otimes \mathbb{1} .
\end{aligned}
$$

For $s=\left(s_{1}, \ldots, s_{d}\right) \in \mathbb{C}^{d}$, put $s^{\prime}=\left(s_{d-f+1}, \ldots, s_{d}, s_{1}, \ldots, s_{d-f}\right)$. For $\Phi_{s}^{\prime} \in$ $V_{s}^{\prime}$ and $g \in G$, put

$$
\mathcal{M}_{s}\left(\Phi_{s}^{\prime}\right)(g)=\int_{\mathrm{M}_{f, d-f}(F)} \Phi_{s}^{\prime}\left(w\left(\begin{array}{cc}
\mathbf{1}_{f} & x \\
0 & \mathbf{1}_{d-f}
\end{array}\right) g\right) d x
$$

where

$$
w=\left(\begin{array}{cc}
0 & \mathbf{1}_{d-f} \\
\mathbf{1}_{f} & 0
\end{array}\right)
$$

and $d x$ is the Haar measure on $\mathrm{M}_{f, d-f}(F)$ such that $\operatorname{vol}\left(\mathrm{M}_{f, d-f}(\mathfrak{o})\right)=1$. This integral is absolutely convergent for $\operatorname{Re}\left(s_{1}\right) \gg \operatorname{Re}\left(s_{2}\right) \gg \cdots \gg \operatorname{Re}\left(s_{d}\right) \gg 0$, has a meromorphic continuation to $\mathbb{C}^{d}$, and defines an intertwining operator

$$
\mathcal{M}_{s}: V_{s}^{\prime} \longrightarrow V_{s^{\prime}}
$$

Then we have $\mathcal{M}_{s} \circ \pi_{s}^{\prime}=\pi_{s^{\prime}} \circ \mathcal{M}_{s}$. By $[17$, Theorem 5.1], we have

$$
\lambda_{s}^{\prime}=\gamma\left(0, \pi_{s, 1}^{\prime} \times \check{\pi}_{s, 2}^{\prime}, \psi\right) \cdot \lambda_{s^{\prime}} \circ \mathcal{M}_{s}
$$

where $\check{\pi}_{s, 2}^{\prime}$ is the contragredient representation of $\pi_{s, 2}^{\prime}$. We remark that $\pi_{s, 2}^{\prime}$ is unramified.

We define a normalized intertwining operator

$$
\mathcal{N}: V^{\prime} \longrightarrow V
$$

by

$$
\mathcal{N}=\lim _{s \rightarrow 0} \gamma\left(0, \pi_{s, 1}^{\prime} \times \check{\pi}_{s, 2}^{\prime}, \psi\right) \mathcal{M}_{s}
$$


Then we have

$$
\mathcal{N} \circ \pi^{\prime}=\pi \circ \mathcal{N}
$$

and

$$
\lambda^{\prime}=\lambda \circ \mathcal{N}
$$

We define an isomorphism

$$
\mathcal{A}_{\omega}: V \longrightarrow V
$$

as vector spaces by

$$
\mathcal{A}_{\omega}=\omega(-1)^{d(d-1) / 2} \cdot \mathcal{N} \circ \mathcal{A}
$$

By Lemma 6.1 and (6.1), we have

$$
\mathcal{A}_{\omega} \circ(\pi \otimes \omega)=\pi \circ \mathcal{A}_{\omega}
$$

LEMMA 6.3. We have

$$
\lambda \circ \mathcal{A}_{\omega}=\lambda .
$$

Proof. By Lemma 6.2 and (6.2), we have

$$
\lambda \circ \mathcal{A}_{\omega}=\omega(-1)^{d(d-1) / 2} \cdot \lambda \circ \mathcal{N} \circ \mathcal{A}=\omega(-1)^{d(d-1) / 2} \cdot \lambda^{\prime} \circ \mathcal{A}=\lambda .
$$

\section{$\S 7$. Calculation of a twisted character}

We retain the notation of Section 6 . In this section, we compute the twisted character $J^{\omega}\left(\pi, f^{G}\right)$ for a certain $f^{G} \in C_{c}^{\infty}(G)$.

Let $K=\mathrm{GL}_{d}(\mathfrak{o})$. Let $I$ be the parahoric subgroup of $G$ given by

$$
I=\left\{g \in K \mid g_{i, j} \in \mathrm{M}_{f}(\mathfrak{p}) \text { if } i>j\right\} .
$$

Here we write $g=\left(g_{i, j}\right)_{1 \leq i, j \leq e}$ with $g_{i, j} \in \mathrm{M}_{f}(F)$. We take a Haar measure $d g$ on $G$ so that $\operatorname{vol}(K)=1$.

Put

$$
\kappa(g)=\omega\left(g_{2,2}\right) \omega^{2}\left(g_{3,3}\right) \cdots \omega^{e-1}\left(g_{e, e}\right)
$$

for $g=\left(g_{i, j}\right)_{1 \leq i, j \leq e} \in I$ with $g_{i, j} \in \mathrm{M}_{f}(F)$. Here we write $\omega\left(g_{i, i}\right)=\omega\left(\operatorname{det} g_{i, i}\right)$ for $g_{i, i} \in \mathrm{GL}_{f}(F)$. Since $\omega$ is trivial on $1+\mathfrak{p}, \kappa$ is a character of $I$. Note that $\kappa(g)=1$ if $g \in I$ is topologically unipotent. We define $f^{G} \in C_{c}^{\infty}(G)$ so that 
- $\operatorname{supp} f^{G}=\eta^{-1} I$,

- $f^{G}\left(\eta^{-1} g\right)=\kappa^{-1} \omega^{-1}(g) \cdot \operatorname{vol}(I)^{-1}$ for $g \in I$,

where

$$
\eta=\left(\begin{array}{cc}
0 & \mathbf{1}_{d-f} \\
\varpi \cdot \mathbf{1}_{f} & 0
\end{array}\right) .
$$

Note that $f^{G}$ does not depend on the choice of $\varpi$.

LEMMA 7.1. We have

$$
f^{G}\left(g_{1} g g_{2}\right)=\kappa^{-1}\left(g_{1}\right) \kappa^{-1} \omega^{-1}\left(g_{2}\right) f^{G}(g)
$$

for $g \in G$ and $g_{1}, g_{2} \in I$.

Proof. Since $\eta I \eta^{-1}=I$, it suffices to show that

$$
\kappa^{-1} \omega^{-1}\left(\eta g \eta^{-1}\right)=\kappa^{-1}(g)
$$

for $g \in I$. We write $\eta g \eta^{-1}=\left(g_{i, j}^{\prime}\right)_{1 \leq i, j \leq e} \in I$ for $g=\left(g_{i, j}\right)_{1 \leq i, j \leq e} \in I$. Then we have

$$
g_{i, i}^{\prime}= \begin{cases}g_{i+1, i+1} & \text { if } 1 \leq i<e \\ g_{1,1} & \text { if } i=e\end{cases}
$$

Hence, we have

$$
\begin{aligned}
\kappa^{-1} \omega^{-1}\left(\eta g \eta^{-1}\right)= & \omega^{-1}\left(g_{3,3}\right) \cdots \omega^{-e+2}\left(g_{e, e}\right) \omega^{-e+1}\left(g_{1,1}\right) \\
& \cdot \omega^{-1}\left(g_{2,2}\right) \cdots \omega^{-1}\left(g_{e, e}\right) \omega^{-1}\left(g_{1,1}\right) \\
= & \omega^{-1}\left(g_{2,2}\right) \omega^{-2}\left(g_{3,3}\right) \cdots \omega^{-e+1}\left(g_{e, e}\right) \\
= & \kappa^{-1}(g) .
\end{aligned}
$$

Let

$$
V_{s}^{\kappa}=\left\{\Phi_{s} \in V_{s} \mid \Phi_{s}\left(g g^{\prime}\right)=\Phi_{s}(g) \kappa\left(g^{\prime}\right) \text { for all } g \in G, g^{\prime} \in I\right\} .
$$

We define $V_{s}^{\kappa \omega}$ and $V_{s}^{\prime, \kappa \omega}$ similarly. We write $V^{\kappa}=V_{0}^{\kappa}, V^{\kappa \omega}=V_{0}^{\kappa \omega}$, and $V^{\prime, \kappa \omega}=V_{0}^{\prime, \kappa \omega}$.

LEMMA 7.2. We have

$$
\operatorname{dim}_{\mathbb{C}} V_{s}^{\kappa}=\operatorname{dim}_{\mathbb{C}} V_{s}^{\kappa \omega}=\operatorname{dim}_{\mathbb{C}} V_{s}^{\prime, \kappa \omega}=1
$$


Proof. We only consider $V_{s}^{\kappa}$. We have an Iwasawa decomposition $G=$ $B_{0} K$. Let $\mathbf{P}^{\prime}$ be the standard parabolic subgroup of $\mathbf{G}$ with Levi factor

$$
\mathbf{L}^{\prime}=\left\{\operatorname{diag}\left(g_{1,1}, \ldots, g_{e, e}\right) \mid g_{i, i} \in \mathrm{GL}_{f}\right\} .
$$

Let $\Omega$ and $\Omega^{\prime}$ be the Weyl groups of $\mathbf{G}$ and $\mathbf{L}^{\prime}$, respectively. We take permutation matrices in $G$ and $L^{\prime}$ as representatives of elements in $\Omega$ and $\Omega^{\prime}$, respectively. Then we have a Bruhat decomposition

$$
K=\coprod_{w^{\prime} \in \Omega / \Omega^{\prime}}\left(B_{0} \cap K\right) w^{\prime} I
$$

Let $\Phi_{s} \in V_{s}$ such that $\operatorname{supp} \Phi_{s} \subset B_{0} w^{\prime} I$. Since $\omega^{e}$ is unramified, the restriction of $\mu_{s}$ to $T_{0} \cap K$ is given by

$$
\underbrace{\mathbb{1} \otimes \cdots \otimes \mathbb{1}}_{f} \otimes \underbrace{\omega \otimes \cdots \otimes \omega}_{f} \otimes \cdots \otimes \underbrace{\omega^{e-1} \otimes \cdots \otimes \omega^{e-1}}_{f} .
$$

Hence, we have

$$
\Phi_{s}\left(w^{\prime} t\right)=\Phi_{s}\left(w^{\prime} t w^{\prime-1} \cdot w^{\prime}\right)=\prod_{i=0}^{e-1} \prod_{j=1}^{f} \omega^{i}\left(t_{w^{\prime}(i f+j)}\right) \cdot \Phi_{s}\left(w^{\prime}\right)
$$

for $t=\operatorname{diag}\left(t_{1}, \ldots, t_{d}\right) \in T_{0} \cap K$. If $\Phi_{s} \in V_{s}^{\kappa}$, then we have

$$
\Phi_{s}\left(w^{\prime} t\right)=\Phi_{s}\left(w^{\prime}\right) \cdot \prod_{i=0}^{e-1} \prod_{j=1}^{f} \omega^{i}\left(t_{i f+j}\right)
$$

for $t=\operatorname{diag}\left(t_{1}, \ldots, t_{d}\right) \in T_{0} \cap K$. Since the restriction of $\omega$ to $\mathfrak{o}^{\times}$is of order $e, \Phi_{s}\left(w^{\prime}\right)=0$ unless $w^{\prime} \in \Omega^{\prime}$.

We define

$$
\Phi_{s}^{\kappa} \in V_{s}^{\kappa}, \quad \Phi_{s}^{\kappa \omega} \in V_{s}^{\kappa \omega}, \quad \Phi_{s}^{\prime, \kappa \omega} \in V_{s}^{\prime, \kappa \omega}
$$

so that

$$
\begin{aligned}
\operatorname{supp} \Phi_{s}^{\kappa} & =B_{0} I, & \Phi_{s}^{\kappa}(1) & =1, \\
\operatorname{supp} \Phi_{s}^{\kappa \omega} & =B_{0} w^{-1} I, & \Phi_{s}^{\kappa \omega}\left(w^{-1}\right) & =1, \\
\operatorname{supp} \Phi_{s}^{\prime, \kappa \omega} & =B_{0} I, & \Phi_{s}^{\prime, \kappa \omega}(1) & =1 .
\end{aligned}
$$


Here

$$
w=\left(\begin{array}{cc}
0 & \mathbf{1}_{d-f} \\
\mathbf{1}_{f} & 0
\end{array}\right) .
$$

We write $\Phi^{\kappa}=\Phi_{0}^{\kappa}, \Phi^{\kappa \omega}=\Phi_{0}^{\kappa \omega}, \Phi^{\prime, \kappa \omega}=\Phi_{0}^{\prime, \kappa \omega}$.

Lemma 7.3. We have

$$
\mathcal{A}\left(\Phi^{\kappa}\right)=\Phi^{\prime, \kappa \omega} .
$$

Proof. We have $\mathcal{A}\left(\Phi^{\kappa}\right) \in V^{\prime, \kappa \omega}$ and

$$
\mathcal{A}\left(\Phi^{\kappa}\right)(1)=\Phi^{\kappa}(1) \omega(1)=1 .
$$

LEMMA 7.4. We have

$$
\mathcal{M}_{s}\left(\Phi_{s}^{\prime, \kappa \omega}\right)=q^{-(e-1) f^{2}} \cdot \Phi_{s^{\prime}}^{\kappa \omega}
$$

Proof. We have $\mathcal{M}_{s}\left(\Phi_{s}^{\prime, \kappa \omega}\right) \in V_{s^{\prime}}^{\kappa \omega}$ and

$$
\begin{aligned}
\mathcal{M}_{s}\left(\Phi_{s}^{\prime, \kappa \omega}\right)\left(w^{-1}\right) & =\int_{\mathrm{M}_{f, d-f}(F)} \Phi_{s}^{\prime, \kappa \omega}\left(w\left(\begin{array}{cc}
\mathbf{1}_{f} & x \\
0 & \mathbf{1}_{d-f}
\end{array}\right) w^{-1}\right) d x \\
& =\int_{\mathrm{M}_{f, d-f}(F)} \Phi_{s}^{\prime, \kappa \omega}\left(\left(\begin{array}{cc}
\mathbf{1}_{d-f} & 0 \\
x & \mathbf{1}_{f}
\end{array}\right)\right) d x
\end{aligned}
$$

Since

$$
\left(\begin{array}{cc}
\mathbf{1}_{d-f} & 0 \\
x & \mathbf{1}_{f}
\end{array}\right) \in B_{0} I
$$

if and only if $x \in \mathrm{M}_{f, d-f}(\mathfrak{p})$, this integral is equal to $\operatorname{vol}\left(\mathrm{M}_{f, d-f}(\mathfrak{p})\right)=$ $q^{-f(d-f)}$.

Let

$$
\lambda(E / F, \psi)=\prod_{i=0}^{f-1} \prod_{j=1}^{e-1} \varepsilon\left(1 / 2, \omega^{i e+j}, \psi\right)
$$

denote the Langlands $\lambda$-factor.

LEMMA 7.5. We have

$$
\lambda(E / F, \psi)^{f}=\omega(-1)^{((e-1) e / 2)[(f+1) / 2]} \lambda(E / F, \psi)^{-1} .
$$


Proof. Note that $\omega^{e}(-1)=1$. If $f$ is even, then we have

$$
\begin{aligned}
\lambda(E / F, \psi) & =\prod_{i=0}^{f / 2-1} \prod_{j=1}^{e-1} \varepsilon\left(1 / 2, \omega^{i e+j}, \psi\right) \varepsilon\left(1 / 2, \omega^{(f-i) e-j}, \psi\right) \\
& =\prod_{i=0}^{f / 2-1} \prod_{j=1}^{e-1} \omega^{i e+j}(-1) \\
& =\prod_{j=1}^{e-1} \omega^{j}(-1)^{f / 2} \\
& =\omega(-1)^{(e-1) e f / 4}
\end{aligned}
$$

In particular, $\lambda(E / F, \psi)= \pm 1$. If $f$ is odd, then we have

$$
\begin{aligned}
\lambda(E / F, \psi)^{2} & =\prod_{i=0}^{f-1} \prod_{j=1}^{e-1} \varepsilon\left(1 / 2, \omega^{i e+j}, \psi\right) \varepsilon\left(1 / 2, \omega^{(f-i) e-j}, \psi\right) \\
& =\prod_{i=0}^{f-1} \prod_{j=1}^{e-1} \omega^{i e+j}(-1) \\
& =\prod_{j=1}^{e-1} \omega^{j}(-1)^{f} \\
& =\prod_{j=1}^{e-1} \omega^{j}(-1) \\
& =\omega(-1)^{(e-1) e / 2} .
\end{aligned}
$$

LEMMA 7.6. We have

$$
\mathcal{N}\left(\Phi^{\prime, \kappa \omega}\right)=q^{-(e-1) f^{2} / 2} \omega(-1)^{((e-1) e / 2)[(f+1) / 2]} \lambda(E / F, \psi)^{-1} \cdot \Phi^{\kappa \omega} .
$$

Proof. We have

$$
\gamma\left(0, \pi_{1}^{\prime} \times \check{\pi}_{2}^{\prime}, \psi\right)=\prod_{i=0}^{f-1} \prod_{j=1}^{e-1} \prod_{k=0}^{f-1} \gamma\left(0, \omega^{i e+j} \cdot \omega^{-k e}, \psi\right) .
$$

Since $\omega$ is of order $e f$, we have

$$
\prod_{i=0}^{f-1} \gamma\left(0, \omega^{i e+j} \cdot \omega^{-k e}, \psi\right)=\prod_{i=0}^{f-1} \gamma\left(0, \omega^{i e+j}, \psi\right)
$$


for fixed $j, k$. Hence, we have

$$
\gamma\left(0, \pi_{1}^{\prime} \times \check{\pi}_{2}^{\prime}, \psi\right)=\prod_{i=0}^{f-1} \prod_{j=1}^{e-1} \gamma\left(0, \omega^{i e+j}, \psi\right)^{f}
$$

Let $0 \leq i \leq f-1$, and let $1 \leq j \leq e-1$. Since $\psi$ is of order zero and $\omega^{i e+j}$ is ramified with conductor 1 , we have

$$
\gamma\left(0, \omega^{i e+j}, \psi\right)=\varepsilon\left(0, \omega^{i e+j}, \psi\right)=q^{1 / 2} \cdot \varepsilon\left(1 / 2, \omega^{i e+j}, \psi\right) .
$$

Thus, we obtain

$$
\gamma\left(0, \pi_{1}^{\prime} \times \check{\pi}_{2}^{\prime}, \psi\right)=q^{(e-1) f^{2} / 2} \prod_{i=0}^{f-1} \prod_{j=1}^{e-1} \varepsilon\left(1 / 2, \omega^{i e+j}, \psi\right)^{f}=q^{(e-1) f^{2} / 2} \lambda(E / F, \psi)^{f} .
$$

Hence, the lemma follows from Lemmas 7.4 and 7.5.

LEMMA 7.7. We have

$$
\pi\left(f^{G}\right) \Phi^{\kappa \omega}=q^{(e-1) f^{2} / 2} \omega(\varpi)^{-d(f-1) / 2} \cdot \Phi^{\kappa} .
$$

Proof. By Lemma 7.1, we have $\pi\left(f^{G}\right) \Phi^{\kappa \omega} \in V^{\kappa}$ and

$$
\begin{aligned}
\pi\left(f^{G}\right) \Phi^{\kappa \omega}(1) & =\int_{G} f^{G}(g) \Phi^{\kappa \omega}(g) d g \\
& =\int_{I} f^{G}\left(\eta^{-1} g\right) \Phi^{\kappa \omega}\left(\eta^{-1} g\right) d g \\
& =f^{G}\left(\eta^{-1}\right) \Phi^{\kappa \omega}\left(\eta^{-1}\right) \cdot \operatorname{vol}(I) .
\end{aligned}
$$

We have $f^{G}\left(\eta^{-1}\right)=\operatorname{vol}(I)^{-1}$ and

$$
\Phi^{\kappa \omega}\left(\eta^{-1}\right)=\Phi^{\kappa \omega}\left(\left(\begin{array}{cc}
\varpi^{-1} \cdot \mathbf{1}_{f} & 0 \\
0 & \mathbf{1}_{d-f}
\end{array}\right) w^{-1}\right)=q^{(e-1) f^{2} / 2} \omega(\varpi)^{-e f(f-1) / 2} .
$$

Proposition 7.8. We have

$$
J^{\omega}\left(\pi, f^{G}\right)=\lambda(E / F, \psi)^{-1} \times \begin{cases}1 & \text { if } e \text { and } f \text { are odd, } \\ \omega(\varpi)^{d / 2} & \text { if } e \text { is odd and } f \text { is even, } \\ \omega(-1)^{e(f-1) / 4} & \text { if } e \text { is even and } f \text { is odd, } \\ \omega(-1)^{e f / 4} \omega(\varpi)^{d / 2} & \text { if } e \text { and } f \text { are even. }\end{cases}
$$


Proof. By Lemma 7.1, we have $\operatorname{Im}\left(\pi\left(f^{G}\right) \circ \mathcal{A}_{\omega}\right) \subset V^{\kappa}$. Since $\operatorname{dim}_{\mathbb{C}} V^{\kappa}=1$ by Lemma 7.2 , we have

$$
J^{\omega}\left(\pi, f^{G}\right)=C,
$$

where $C \in \mathbb{C}$ is a constant such that $\pi\left(f^{G}\right) \mathcal{A}_{\omega}\left(\Phi^{\kappa}\right)=C \cdot \Phi^{\kappa}$. It remains to compute $C=\pi\left(f^{G}\right) \mathcal{A}_{\omega}\left(\Phi^{\kappa}\right)(1)$. By Lemmas 7.3, 7.6, and 7.7, we have

$$
\begin{aligned}
\pi\left(f^{G}\right. & \mathcal{A}_{\omega}\left(\Phi^{\kappa}\right)(1) \\
& =\omega(-1)^{d(d-1) / 2} \pi\left(f^{G}\right) \mathcal{N}\left(\mathcal{A}\left(\Phi^{\kappa}\right)\right)(1) \\
& =\omega(-1)^{d(d-1) / 2} \pi\left(f^{G}\right) \mathcal{N}\left(\Phi^{\prime, \kappa \omega}\right)(1) \\
& =q^{-(e-1) f^{2} / 2} \omega(-1)^{d(d-1) / 2+((e-1) e / 2)[(f+1) / 2]} \lambda(E / F, \psi)^{-1} \pi\left(f^{G}\right) \Phi^{\kappa \omega}(1) \\
& =\omega(-1)^{d(d-1) / 2+((e-1) e / 2)[(f+1) / 2]} \omega(\varpi)^{-d(f-1) / 2} \lambda(E / F, \psi)^{-1} \Phi^{\kappa}(1) \\
& =\omega(-1)^{d(d-1) / 2+((e-1) e / 2)[(f+1) / 2]} \omega(\varpi)^{-d(f-1) / 2} \lambda(E / F, \psi)^{-1}
\end{aligned}
$$

Since $\omega^{e}(-1)=1$, we have

$$
\omega(-1)^{d(d-1) / 2}= \begin{cases}1 & \text { if } e \text { and } f \text { are odd } \\ 1 & \text { if } e \text { is odd and } f \text { is even } \\ \omega(-1)^{e / 2} & \text { if } e \text { is even and } f \text { is odd } \\ 1 & \text { if } e \text { and } f \text { are even }\end{cases}
$$

and

$$
\omega(-1)^{(e(e-1) / 2)[(f+1) / 2]}= \begin{cases}1 & \text { if } e \text { and } f \text { are odd } \\ 1 & \text { if } e \text { is odd and } f \text { is even, } \\ \omega(-1)^{e(f+1) / 4} & \text { if } e \text { is even and } f \text { is odd } \\ \omega(-1)^{e f / 4} & \text { if } e \text { and } f \text { are even }\end{cases}
$$

Since $\omega^{d}(\varpi)=1$, we have $\omega(\varpi)^{-d(f-1) / 2}=1$ if $f$ is odd. If $f$ is even, then $\omega(\varpi)^{d / 2}= \pm 1$, so that $\omega(\varpi)^{-d(f-1) / 2}=\omega(\varpi)^{d / 2}$. This completes the proof.

\section{$\S 8$. Calculation of a twisted orbital integral}

We retain the notation of Sections 6 and 7. In this section, we compute the twisted orbital integral $J^{\omega}\left(\gamma, f^{G}\right)$ for $f^{G} \in C_{c}^{\infty}(G)$ defined in Section 7 . 
For each $0 \leq i<d$, let $\mathcal{L}_{i, G}$ be the lattice of $F^{d}$ given by

$$
\mathcal{L}_{i, G}=\underbrace{\mathfrak{a} \oplus \cdots \oplus \mathfrak{o}}_{d-i} \oplus \underbrace{\mathfrak{p} \oplus \cdots \oplus \mathfrak{p}}_{i} .
$$

For each integer $i=j d+k$ with $0 \leq k<d$, let $\mathcal{L}_{i, G}=\varpi^{j} \mathcal{L}_{k, G}$. Then we have

$$
I=\left\{g \in G \mid g \mathcal{L}_{i f, G} \subset \mathcal{L}_{i f, G} \text { for all } i\right\} .
$$

Fix an isomorphism $\phi: E \rightarrow F^{d}$ as vector spaces over $F$ such that

$$
\phi\left(\mathfrak{p}_{E}^{i}\right)=\mathcal{L}_{i f, G}
$$

for all $i$. Let $\iota: \mathbf{H} \rightarrow \mathbf{G}$ be the embedding induced by $\phi$. Then we have

$$
\iota(H) \cap I=\iota\left(\mathfrak{o}_{E}^{\times}\right) .
$$

We identify $H$ with the image in $G$ and suppress $\iota$ from the notation.

Let $\gamma \in G$ be a regular semisimple element, and let $\mathbf{T}$ be the centralizer of $\gamma$ in $\mathbf{G}$. Then $J^{\omega}\left(\gamma, f^{G}\right)=0$ unless $\gamma$ is conjugate to an element in $H$. If $\gamma \in H$ is $G$-regular, then $\mathbf{T}=\mathbf{H}$.

Lemma 8.1. Let $\gamma \in H$ be a $G$-regular semisimple element. If $J^{\omega}\left(\gamma, f^{G}\right) \neq$ 0 , then we have

$$
\operatorname{ord}_{E} \gamma=-1 \text {. }
$$

Proof. Then, since supp $f^{G}=\eta^{-1} I$, we have $J^{\omega}\left(\gamma, f^{G}\right)=0$ unless $\operatorname{ord}_{F} \operatorname{det} \gamma=-f$.

Let $\gamma \in H$ be a $G$-regular semisimple element such that $\operatorname{ord}_{E} \gamma=-1$. By [12] and [19, section VII.1], there exist $\delta, \gamma^{\prime} \in E^{\times}$such that

- $\gamma=\delta \gamma^{\prime}$,

- $\gamma^{\prime} \in 1+\mathfrak{p}_{E}$,

- $\delta$ is $F(\delta) / F$-cuspidal (see [19, section VI.2]).

Put $F^{\prime}=F(\delta)$. Note that $F^{\prime}$ does not depend on the choice of $\delta, \gamma^{\prime}$.

Lemma 8.2. We have $\operatorname{ord}_{F^{\prime}} \delta=-1$. Moreover, $E / F^{\prime}$ is unramified.

Proof. Let $e^{\prime}$ be the ramification index of $E / F^{\prime}$. Then we have

$$
\operatorname{ord}_{E} \gamma=\operatorname{ord}_{E} \delta=e^{\prime} \operatorname{ord}_{F^{\prime}} \delta \text {. }
$$

Hence, $e^{\prime}=1$. 
Note that the ramification index of $F^{\prime} / F$ is $e$. Let $f^{\prime}$ be the residual degree of $F^{\prime} / F$. Put $d^{\prime}=d / e f^{\prime}$. Let $\mathbf{G}^{\prime}=\operatorname{Res}_{F^{\prime} / F} \mathrm{GL}_{d^{\prime}}$, and let $K^{\prime}=\mathrm{GL}_{d^{\prime}}\left(\mathfrak{o}_{F^{\prime}}\right)$. We take a Haar measure $d g^{\prime}$ on $G^{\prime}$ so that $\operatorname{vol}\left(K^{\prime}\right)=1$. Fix an isomorphism $\phi^{\prime}: F^{\prime d^{\prime}} \rightarrow F^{d}$ such that $\phi^{\prime}\left(\mathcal{L}_{i, G^{\prime}}\right)=\mathcal{L}_{i f^{\prime}, G}$ for all $i$. Let $\iota: \mathbf{G}^{\prime} \rightarrow \mathbf{G}$ be the embedding induced by $\phi^{\prime}$. Then we have

$$
\iota\left(G^{\prime}\right) \cap I=\iota\left(K^{\prime}\right), \quad \iota\left(G^{\prime}\right) \cap \eta^{-1} I=\iota\left(\delta K^{\prime}\right) .
$$

For simplicity, we will suppress $\iota$ from the notation. We may also regard $H$ as a subgroup of $G^{\prime}$ via $\phi$ and $\phi^{\prime}$.

Lemma 8.3. Let $g \in G$ such that $g^{-1} \gamma g \in \eta^{-1} I$. Then we have

$$
g \in G^{\prime} I
$$

Proof. The lemma follows from [19, lemme VI.3].

Lemma 8.4. We have $\operatorname{supp} f^{G} \cap G^{\prime}=\delta K^{\prime}$. Thus, let $g \in G^{\prime}$ such that $g^{-1} \gamma^{\prime} g \in K^{\prime}$. Then we have

$$
f^{G}\left(g^{-1} \gamma g\right)=f^{G}(\delta)
$$

Proof. The first assertion is obvious. Since $\gamma^{\prime} \in 1+\mathfrak{p}_{E}, g^{-1} \gamma^{\prime} g \in K^{\prime}$ is topologically unipotent, and hence $\kappa^{-1} \omega^{-1}\left(g^{-1} \gamma^{\prime} g\right)=1$. Thus, we obtain

$$
f^{G}\left(g^{-1} \gamma g\right)=f^{G}\left(\delta g^{-1} \gamma^{\prime} g\right)=f^{G}(\delta) \kappa^{-1} \omega^{-1}\left(g^{-1} \gamma^{\prime} g\right)=f^{G}(\delta) .
$$

Let $\omega^{\prime}=\omega \circ \mathrm{N}_{F^{\prime} / F}$ be an unramified character of $F^{\prime \times}$ of order $d^{\prime}$ associated to $E / F^{\prime}$ by class field theory. Let $\mathbf{1}_{K^{\prime}} \in C_{c}^{\infty}\left(G^{\prime}\right)$ be the characteristic function of $K^{\prime}$. Put

$$
J^{\omega^{\prime}}\left(\gamma^{\prime}, \mathbf{1}_{K^{\prime}}\right)=\int_{T \backslash G^{\prime}} \omega^{\prime}\left(g^{\prime}\right) \mathbf{1}_{K^{\prime}}\left(g^{\prime-1} \gamma^{\prime} g^{\prime}\right) d g^{\prime} .
$$

Note that $\mathbf{T}$ is also the centralizer of $\gamma^{\prime}$ in $\mathbf{G}^{\prime}$.

LEMmA 8.5. Let $\gamma \in H$ be a G-regular semisimple element such that $\operatorname{ord}_{E} \gamma=-1$. Let $\delta, \gamma^{\prime} \in H$ as above. Then we have

$$
J^{\omega}\left(\gamma, f^{G}\right)=\operatorname{vol}(I) f^{G}(\delta) \cdot J^{\omega^{\prime}}\left(\gamma^{\prime}, \mathbf{1}_{K^{\prime}}\right) .
$$


Proof. The lemma follows from [19, lemme VI.4] with slight modifications. We include the proof for the sake of completeness.

Note that

$$
\omega(g) f^{G}\left(g^{-1} \gamma g\right)=\omega(g) \kappa(g) \kappa^{-1} \omega^{-1}(g) f^{G}(\gamma)=f^{G}(\gamma)
$$

for $g \in I$. By Lemma 8.3, we have

$$
J^{\omega}\left(\gamma, f^{G}\right)=\sum_{g \in \Gamma} \operatorname{vol}(T \backslash T g I) \omega(\iota(g)) f^{G}\left(g^{-1} \gamma g\right) .
$$

Here $\Gamma$ is a set of representatives of equivalence classes of $G^{\prime}$ for the equivalence $g_{1} \sim g_{2}$ if and only if $g_{1} \in T g_{2} I$. Then $\Gamma$ is also a set of representatives of $T \backslash G^{\prime} / K^{\prime}$. As in the proof of [19, lemme VI.4], we have $\operatorname{vol}(T \backslash T g I)=\operatorname{vol}(I) \operatorname{vol}\left(T \backslash T g K^{\prime}\right)$ and $\omega(\iota(g))=\omega^{\prime}(g)$ for $g \in \Gamma$. By Lemma 8.4, $f^{G}\left(g^{-1} \gamma g\right)=0$ for $g \in \Gamma$ unless $g^{-1} \gamma^{\prime} g \in K^{\prime}$, and hence

$$
\begin{aligned}
J^{\omega}\left(\gamma, f^{G}\right) & =\operatorname{vol}(I) \sum_{g \in \Gamma} \operatorname{vol}\left(T \backslash T g K^{\prime}\right) \omega^{\prime}(g) f^{G}\left(g^{-1} \gamma g\right) \mathbf{1}_{K^{\prime}}\left(g^{-1} \gamma^{\prime} g\right) \\
& =\operatorname{vol}(I) f^{G}(\delta) \sum_{g \in \Gamma} \operatorname{vol}\left(T \backslash T g K^{\prime}\right) \omega^{\prime}(g) \mathbf{1}_{K^{\prime}}\left(g^{-1} \gamma^{\prime} g\right) \\
& =\operatorname{vol}(I) f^{G}(\delta) \cdot J^{\omega^{\prime}}\left(\gamma^{\prime}, \mathbf{1}_{K^{\prime}}\right) .
\end{aligned}
$$

\section{§9. Calculation of the transfer factor}

Let $\mathbf{G}=\mathrm{GL}_{d}$, and let $\mathbf{H}=\operatorname{Res}_{E / F} \mathbb{G}_{m}$, where $E$ is a tamely ramified cyclic extension of $F$ of degree $d$. Let $(\mathbf{H}, \mathcal{H}, s, \xi)=\left(\operatorname{Res}_{E / F} \mathbb{G}_{m},{ }^{L} H_{\mathbf{m}}, s_{\mathbf{m}}, \xi_{\mathbf{m}}\right)$ be the endoscopic datum for $(\mathbf{G}, 1, \mathbf{a})$ with $\mathbf{m}=(1)$ as in Section 2. In this section, we compute the transfer factor $\Delta(\gamma, \iota(\gamma))$ for $G$-regular semisimple elements $\gamma \in H$, where $\iota: \mathbf{H} \rightarrow \mathbf{G}$ is the embedding defined in Section 8 .

Let $e$ and $f$ be the ramification index and the residual degree of $E / F$, respectively. Let $\omega$ be the character of $F^{\times}$of order $d$ associated to a. Let $\sigma$ be the generator of $\operatorname{Gal}(E / F)$ as in $\left[11\right.$, Section 10]. Let $\eta_{E}$ be the unramified quadratic character of $E^{\times}$. If $d$ is even, let $\sigma_{+}=\sigma^{d / 2}$, and let $E_{+}$be the subfield of $E$ corresponding to the subgroup $\left\{1, \sigma_{+}\right\}$of $\operatorname{Gal}(E / F)$. Let $\omega_{+}=$ $\omega \circ \mathrm{N}_{E_{+} / F}$ be the quadratic character of $E_{+}^{\times}$corresponding to $E / E_{+}$by class field theory. If $e$ is odd and $f$ is even, then $E / E_{+}$is unramified and the restriction of $\eta_{E}$ to $E_{+}^{\times}$is $\omega_{+}$.

We fix a primitive $\left(q^{f}-1\right)$ th root of unity $\zeta$ in $E$. We choose $\varpi_{E}$ and $\varpi_{F}$ so that $\varpi_{E}^{e}=\varpi_{F} \zeta^{a}$ with some integer $a$. If $e$ is odd and $f$ is even, then we 
may assume that $\varpi_{E} \in E_{+}$since the ramification index of $E_{+} / F$ is $e$ and $E / E_{+}$is unramified. Then we have

$$
\sigma_{+}\left(\varpi_{E}\right)= \begin{cases}\varpi_{E} & \text { if } e \text { is odd and } f \text { is even } \\ -\varpi_{E} & \text { if } e \text { is even. }\end{cases}
$$

We take $\left\{\varpi_{E}^{i} \zeta^{j} \mid 0 \leq i<e, 0 \leq j<f\right\}$ as a basis of $\mathfrak{o}_{E}$ over $\mathfrak{o}_{F}$. Put

$$
\vec{u}=\left(\zeta^{f-1}, \zeta^{f-2}, \ldots, \zeta, 1\right) \in E^{f}
$$

and

$$
\vec{v}=\left(\varpi_{E}^{e-1} \cdot \vec{u}, \varpi_{E}^{e-2} \cdot \vec{u}, \ldots, \varpi_{E} \cdot \vec{u}, \vec{u}\right) \in E^{d} .
$$

We may assume that the embedding $\iota: \mathbf{H} \rightarrow \mathbf{G}$ is given by

$$
x \cdot \vec{v}=\vec{v} \cdot \iota(x)
$$

for $x \in H$. Put

$$
g=\left(\begin{array}{c}
\vec{v} \\
\sigma(\vec{v}) \\
\vdots \\
\sigma^{d-1}(\vec{v})
\end{array}\right) \in \mathrm{GL}_{d}(E)
$$

Then we have

$$
g \iota(x) g^{-1}=\operatorname{diag}\left(x, \sigma(x), \sigma^{2}(x), \ldots, \sigma^{d-1}(x)\right)
$$

for $x \in H$.

We will compute $\operatorname{det} g$.

Lemma 9.1. Let $\mathbf{x}_{i}=\operatorname{diag}\left(x_{(i-1) f+1}, x_{(i-1) f+2}, \ldots, x_{i f}\right)$. Then we have

$$
\operatorname{det}\left(\begin{array}{cccc}
\mathbf{1}_{f} & \mathbf{1}_{f} & \cdots & \mathbf{1}_{f} \\
\mathbf{x}_{1} & \mathbf{x}_{2} & \cdots & \mathbf{x}_{e} \\
\mathbf{x}_{1}^{2} & \mathbf{x}_{2}^{2} & \cdots & \mathbf{x}_{e}^{2} \\
\vdots & \vdots & \ddots & \vdots \\
\mathbf{x}_{1}^{e-1} & \mathbf{x}_{2}^{e-1} & \cdots & \mathbf{x}_{e}^{e-1}
\end{array}\right)=\prod_{k=1}^{f} \prod_{0 \leq i<j \leq e-1}\left(x_{j f+k}-x_{i f+k}\right)
$$

Proof. See the Vandermonde determinant. 
Put

$$
\mathbf{u}=\left(\begin{array}{c}
\vec{u} \\
\sigma(\vec{u}) \\
\vdots \\
\sigma^{f-1}(\vec{u})
\end{array}\right) \in \mathrm{GL}_{f}(E) .
$$

Since $\sigma^{f}(\vec{u})=\vec{u}$, we have $g=g^{\prime} g^{\prime \prime}$, where $g^{\prime}=\left(g_{i, j}^{\prime}\right)_{1 \leq i, j \leq e}$, with $g_{i, j}^{\prime}=\operatorname{diag}\left(\sigma^{(i-1) f}\left(\varpi_{E}\right)^{e-j}, \sigma^{(i-1) f+1}\left(\varpi_{E}\right)^{e-j}, \ldots, \sigma^{i f-1}\left(\varpi_{E}\right)^{e-j}\right) \in \mathrm{M}_{f}(E)$, and $g^{\prime \prime}=\operatorname{diag}(\mathbf{u}, \mathbf{u}, \ldots, \mathbf{u})$. We have

$$
\begin{aligned}
\operatorname{det} g^{\prime} & =(-1)^{(e-1) e f / 2} \prod_{k=1}^{f} \prod_{0 \leq i<j \leq e-1}\left(\sigma^{j f+k-1}\left(\varpi_{E}\right)-\sigma^{i f+k-1}\left(\varpi_{E}\right)\right) \\
& =\prod_{k=1}^{f} \prod_{0 \leq i<j \leq e-1}\left(\sigma^{i f+k-1}\left(\varpi_{E}\right)-\sigma^{j f+k-1}\left(\varpi_{E}\right)\right)
\end{aligned}
$$

and

$$
\begin{aligned}
\operatorname{det} g^{\prime \prime} & =\left((-1)^{(f-1) f / 2} \prod_{1 \leq i<j \leq f}\left(\sigma^{j-1}(\zeta)-\sigma^{i-1}(\zeta)\right)\right)^{e} \\
& =\left(\prod_{1 \leq i<j \leq f}\left(\sigma^{i-1}(\zeta)-\sigma^{j-1}(\zeta)\right)\right)^{e} .
\end{aligned}
$$

Hence, we have

$$
\begin{aligned}
\operatorname{det} g= & \prod_{k=1}^{f} \prod_{0 \leq i<j \leq e-1}\left(\sigma^{i f+k-1}\left(\varpi_{E}\right)-\sigma^{j f+k-1}\left(\varpi_{E}\right)\right) \\
& \times\left(\prod_{1 \leq i<j \leq f}\left(\sigma^{i-1}(\zeta)-\sigma^{j-1}(\zeta)\right)\right)^{e}
\end{aligned}
$$

We define $h \in \mathrm{GL}_{d}(E)$ so that

$$
h^{-1}=\left(\begin{array}{c}
b_{1} \cdot \vec{v} \\
b_{2} \cdot \sigma(\vec{v}) \\
\vdots \\
b_{d} \cdot \sigma^{d-1}(\vec{v})
\end{array}\right),
$$


where

$$
\begin{aligned}
b_{j f+k}= & \prod_{0 \leq i<j}\left(\sigma^{i f+k-1}\left(\varpi_{E}\right)-\sigma^{j f+k-1}\left(\varpi_{E}\right)\right)^{-1} \\
& \times \prod_{1 \leq i<k}\left(\sigma^{i-1}(\zeta)-\sigma^{k-1}(\zeta)\right)^{-1}
\end{aligned}
$$

for $0 \leq j \leq e-1,1 \leq k \leq f$. Then we have $\operatorname{det} h=1$; that is, $h \in \mathbf{G}_{\mathrm{SC}}(E)$ and

$$
h^{-1} \iota(x) h=\operatorname{diag}\left(x, \sigma(x), \sigma^{2}(x), \ldots, \sigma^{d-1}(x)\right)
$$

for $x \in H$.

Let $\mathbf{T}_{H}=\mathbf{H}$, and let $\mathbf{T}=\iota\left(\mathbf{T}_{H}\right)$. We choose an admissible embedding $\mathbf{T}_{H} \rightarrow \mathbf{T}$ induced by $\iota$. Then we have

$$
\Delta_{\mathrm{III}_{1}}(\gamma, \iota(\gamma))=1
$$

Let $\alpha_{i, j}$ be the root of $\mathbf{T}$ in $\mathbf{G}$ corresponding to the coroot $\mathbf{t}_{i, i} \mathbf{t}_{j, j}^{-1}$ of $\mathcal{T}$ in $\hat{G}$. Here $\mathbf{t}_{i, i}$ is the cocharacter of $\mathcal{T}$ given by $\mathbf{t}_{i, i}(z)=\operatorname{diag}(1, \ldots, 1, z, 1, \ldots, 1)$ for $z \in \mathbb{C}^{\times}$. Then $\alpha_{i, j}$ is symmetric (resp., asymmetric) if and only if $|i-j|=d / 2$ (resp., $|i-j| \neq d / 2$ ). We choose $a$-data $\left\{a_{\alpha}\right\}$ and $\chi$-data $\left\{\chi_{\alpha}\right\}$ as follows. For simplicity, we write $a_{i, j}=a_{\alpha_{i, j}}$ and $\chi_{i, j}=\chi_{\alpha_{i, j}}$.

We require that $\sigma\left(a_{i, j}\right)=a_{i+1, j+1}$ and $a_{j, i}=-a_{i, j}$. Put

$$
a_{1, j f+k}= \begin{cases}\nu\left(\sigma^{j f}\left(\varpi_{E}\right)-\varpi_{E}\right) & \text { if } k=1, \\ \sigma^{k-1}(\zeta)-\zeta & \text { if } k \neq 1,\end{cases}
$$

where

$$
\nu=\prod_{1 \leq i<f}\left(\sigma^{i}(\zeta)-\zeta\right)^{-1}
$$

Note that $\sigma^{f}(\nu)=\nu$.

LEMMA 9.2. We have

$$
\sigma^{d+1-i}\left(a_{1, i}\right)=-a_{1, d+2-i}
$$

for $1<i \leq d / 2+1$. In particular, $\left\{a_{1, i}\right\}$ determines a-data $\left\{a_{\alpha}\right\}$ uniquely. 
Proof. Note that $i \equiv 1 \bmod f$ if and only if $d+2-i \equiv 1 \bmod f$. Let $i=j f+k$. If $k=1$, then we have

$$
\begin{aligned}
\sigma^{d+1-i}\left(a_{1, i}\right) & =\sigma^{d-j f}\left(a_{1, j f+1}\right)=\nu\left(\varpi_{E}-\sigma^{d-j f}\left(\varpi_{E}\right)\right) \\
& =-a_{1, d-j f+1}=-a_{1, d+2-i} .
\end{aligned}
$$

If $k \neq 1$, then we have $d+2-i=(d-j f-f)+(f+2-k)$ and

$$
\sigma^{d+1-i}\left(a_{1, i}\right)=\sigma^{d-j f-k+1}\left(a_{1, j f+k}\right)=\zeta-\sigma^{f-k+1}(\zeta)=-a_{1, d+2-i}
$$

Put $\chi_{1, i}=\mathbb{1}$ for $1<i<d / 2+1$. If $d$ is even, let $\chi_{1, d / 2+1}$ be a character of $E^{\times}$whose restriction to $E_{+}^{\times}$is $\omega_{+}$. Note that $\chi_{1, d / 2+1} \circ \sigma_{+}=\chi_{1, d / 2+1}^{-1}$. If $e$ is odd and $f$ is even, then we can take $\chi_{1, d / 2+1}=\eta_{E}$. Then $\left\{\chi_{1, i}\right\}$ determines $\chi$-data $\left\{\chi_{\alpha}\right\}$ uniquely.

LEMMA 9.3. If e and $f$ are odd, then we have

$$
\Delta_{\mathrm{II}}(\gamma, \iota(\gamma)) \Delta_{\mathrm{III}_{2}}(\gamma, \iota(\gamma))=1
$$

If $e$ is odd and $f$ is even, then we have

$$
\Delta_{\mathrm{II}}(\gamma, \iota(\gamma)) \Delta_{\mathrm{III}_{2}}(\gamma, \iota(\gamma))=\eta_{E}\left(\gamma-\sigma_{+}(\gamma)\right)
$$

If $e$ is even and $f$ is odd, then we have

$$
\Delta_{\mathrm{II}}(\gamma, \iota(\gamma)) \Delta_{\mathrm{III}_{2}}(\gamma, \iota(\gamma))=\omega(-1)^{e(f-1) / 4} \cdot \omega_{+}\left(\frac{\gamma-\sigma_{+}(\gamma)}{2 \varpi_{E}^{-1}}\right) .
$$

If $e$ and $f$ are even, then we have

$$
\Delta_{\mathrm{II}}(\gamma, \iota(\gamma)) \Delta_{\mathrm{III}_{2}}(\gamma, \iota(\gamma))=\omega_{+}\left(\frac{\gamma-\sigma_{+}(\gamma)}{2 \varpi_{E}^{-1}\left(\sigma^{f / 2}(\zeta)-\zeta\right)}\right)
$$

Proof. We only consider the case when $d$ is even. By [11, Appendix A], we have

$$
\Delta_{\mathrm{II}}(\gamma, \iota(\gamma))=\chi_{1, d / 2+1}\left(\frac{\alpha_{1, d / 2+1}(\gamma)-1}{a_{1, d / 2+1}}\right)=\chi_{1, d / 2+1}\left(\frac{\gamma \sigma_{+}(\gamma)^{-1}-1}{a_{1, d / 2+1}}\right)
$$

and

$$
\Delta_{\mathrm{III}_{2}}(\gamma, \iota(\gamma))=\chi_{1, d / 2+1}(\gamma)^{-1}
$$

Since $\sigma_{+}\left(a_{1, d / 2+1}\right)=-a_{1, d / 2+1}$, we have

$$
\Delta_{\mathrm{II}}(\gamma, \iota(\gamma)) \Delta_{\mathrm{III}_{1}}(\gamma, \iota(\gamma))=\chi_{1, d / 2+1}\left(\frac{\sigma_{+}(\gamma)^{-1}-\gamma^{-1}}{a_{1, d / 2+1}}\right)=\omega_{+}\left(\frac{\gamma-\sigma_{+}(\gamma)}{a_{1, d / 2+1}}\right)
$$


Note that $\omega_{+}\left(x \sigma_{+}(x)\right)=1$ for $x \in E^{\times}$.

If $e$ is odd, then $f$ is even and

$$
a_{1, d / 2+1}=a_{1,(e-1) f / 2+f / 2+1}=\sigma^{f / 2}(\zeta)-\zeta \in \mathfrak{o}_{E}^{\times},
$$

so that

$$
\chi_{1, d / 2+1}\left(a_{1, d / 2+1}\right)=\eta_{E}\left(\sigma^{f / 2}(\zeta)-\zeta\right)=1 .
$$

Assume that $e$ is even. Then we have

$$
a_{1, d / 2+1}=a_{1, e f / 2+1}=\nu\left(\sigma^{e f / 2}\left(\varpi_{E}\right)-\varpi_{E}\right)=-2 \varpi_{E} \cdot \nu
$$

and

$$
\chi_{1, d / 2+1}\left(-\varpi_{E}\right)=\chi_{1, d / 2+1}\left(\sigma_{+}\left(\varpi_{E}\right)\right)=\chi_{1, d / 2+1}\left(\varpi_{E}^{-1}\right) .
$$

Since $\sigma^{i}(\zeta)-\zeta \in E_{+}^{\times}$and

$$
\omega_{+}(\sigma(x))=\omega\left(\mathrm{N}_{E_{+} / F}(\sigma(x))\right)=\omega\left(\mathrm{N}_{E_{+} / F}(x)\right)=\omega_{+}(x)
$$

for $x \in E_{+}^{\times}$, we have

$$
\begin{aligned}
\omega_{+}\left(\sigma^{i}(\zeta)-\zeta\right) & =\omega_{+}\left(\sigma^{f-i}\left(\sigma^{i}(\zeta)-\zeta\right)\right)=\omega_{+}\left(\zeta-\sigma^{f-i}(\zeta)\right) \\
& =\omega(-1)^{d / 2} \omega_{+}\left(\sigma^{f-i}(\zeta)-\zeta\right)
\end{aligned}
$$

If $f$ is odd, then we have

$$
\begin{aligned}
\omega_{+}(\nu) & =\prod_{1 \leq i \leq(f-1) / 2} \omega_{+}\left(\sigma^{i}(\zeta)-\zeta\right)^{-1} \cdot \prod_{(f+1) / 2 \leq i \leq f-1} \omega_{+}\left(\sigma^{i}(\zeta)-\zeta\right)^{-1} \\
& =\omega(-1)^{d(f-1) / 4} \prod_{1 \leq i \leq(f-1) / 2} \omega_{+}\left(\sigma^{i}(\zeta)-\zeta\right)^{-2} \\
& =\omega(-1)^{d(f-1) / 4} \\
& =\omega(-1)^{e(f-1) / 4}
\end{aligned}
$$

If $f$ is even, then we have

$$
\begin{aligned}
\omega_{+}(\nu)= & \prod_{1 \leq i \leq f / 2-1} \omega_{+}\left(\sigma^{i}(\zeta)-\zeta\right)^{-1} \cdot \omega_{+}\left(\sigma^{f / 2}(\zeta)-\zeta\right)^{-1} \\
& \cdot \prod_{f / 2+1 \leq i \leq f-1} \omega_{+}\left(\sigma^{i}(\zeta)-\zeta\right)^{-1}
\end{aligned}
$$




$$
\begin{aligned}
& =\omega(-1)^{d(f-2) / 4} \prod_{1 \leq i \leq f / 2-1} \omega_{+}\left(\sigma^{i}(\zeta)-\zeta\right)^{-2} \cdot \omega_{+}\left(\sigma^{f / 2}(\zeta)-\zeta\right)^{-1} \\
& =\omega(-1)^{d(f-2) / 4} \cdot \omega_{+}\left(\sigma^{f / 2}(\zeta)-\zeta\right) \\
& =\omega_{+}\left(\sigma^{f / 2}(\zeta)-\zeta\right)
\end{aligned}
$$

This completes the proof.

LEMMA 9.4. We have

$$
\Delta_{\mathrm{I}}(\gamma, \iota(\gamma))=1
$$

Proof. Let $h \in \mathbf{G}_{\mathrm{SC}}(E)$ be given by (9.1). Let $m\left(\sigma_{T}\right)=x\left(\sigma_{T}\right) n\left(\omega_{T}(\sigma)\right)$ be as in [15, Section 2.3]. Then we have

$$
x\left(\sigma_{T}\right)=\operatorname{diag}\left(\prod_{1<i \leq d} a_{1, i}, a_{1,2}^{-1}, a_{1,3}^{-1}, \ldots, a_{1, d}^{-1}\right)
$$

and

$$
n\left(\omega_{T}(\sigma)\right)=\left(\begin{array}{cc}
0 & 1 \\
-\mathbf{1}_{d-1} & 0
\end{array}\right)
$$

Hence, we have

$$
m\left(\sigma_{T}\right) \sigma\left(h^{-1}\right)=x\left(\sigma_{T}\right) \cdot\left(\begin{array}{c}
\sigma\left(b_{d}\right) \cdot \vec{v} \\
-\sigma\left(b_{1}\right) \cdot \sigma(\vec{v}) \\
\vdots \\
-\sigma\left(b_{d-1}\right) \cdot \sigma^{d-1}(\vec{v})
\end{array}\right)=\left(\begin{array}{c}
\prod_{1<i \leq d} a_{1, i} \cdot \sigma\left(b_{d}\right) \cdot \vec{v} \\
-a_{1,2}^{-1} \sigma\left(b_{1}\right) \cdot \sigma(\vec{v}) \\
\vdots \\
-a_{1, d}^{-1} \sigma\left(b_{d-1}\right) \cdot \sigma^{d-1}(\vec{v})
\end{array}\right) .
$$

Let $i=j f+k \geq 2$ with $0 \leq j \leq e-1,1 \leq k \leq f$. If $k=1$, then we have

$$
\begin{aligned}
-a_{1, i}^{-1} \sigma\left(b_{i-1}\right)= & -a_{1, j f+1}^{-1} \sigma\left(b_{(j-1) f+f}\right) \\
= & \nu^{-1}\left(\varpi_{E}-\sigma^{j f}\left(\varpi_{E}\right)\right)^{-1} \prod_{0 \leq i<j-1}\left(\sigma^{(i+1) f}\left(\varpi_{E}\right)-\sigma^{j f}\left(\varpi_{E}\right)\right)^{-1} \\
& \times \prod_{1 \leq i<f}\left(\sigma^{i}(\zeta)-\zeta\right)^{-1} \\
= & \prod_{0 \leq i<j}\left(\sigma^{i f}\left(\varpi_{E}\right)-\sigma^{j f}\left(\varpi_{E}\right)\right)^{-1} \\
= & b_{j f+1} \\
= & b_{i} .
\end{aligned}
$$


If $k \neq 1$, then we have

$$
\begin{aligned}
-a_{1, i}^{-1} \sigma\left(b_{i-1}\right)= & -a_{1, j f+k}^{-1} \sigma\left(b_{j f+k-1}\right) \\
= & \left(\zeta-\sigma^{k-1}(\zeta)\right)^{-1} \prod_{0 \leq i<j}\left(\sigma^{i f+k-1}\left(\varpi_{E}\right)-\sigma^{j f+k-1}\left(\varpi_{E}\right)\right)^{-1} \\
& \times \prod_{1 \leq i<k-1}\left(\sigma^{i}(\zeta)-\sigma^{k-1}(\zeta)\right)^{-1} \\
= & \prod_{0 \leq i<j}\left(\sigma^{i f+k-1}\left(\varpi_{E}\right)-\sigma^{j f+k-1}\left(\varpi_{E}\right)\right)^{-1} \\
& \times \prod_{1 \leq i<k}\left(\sigma^{i-1}(\zeta)-\sigma^{k-1}(\zeta)\right)^{-1} \\
= & b_{j f+k} \\
= & b_{i} .
\end{aligned}
$$

Since

$$
\begin{aligned}
\prod_{2 \leq i \leq d} a_{1, i} & =\nu^{e-1} \prod_{1 \leq i \leq e-1}\left(\sigma^{i f}\left(\varpi_{E}\right)-\varpi_{E}\right) \cdot\left(\prod_{1<k \leq f}\left(\sigma^{k-1}(\zeta)-\zeta\right)\right)^{e} \\
& =\nu^{-1} \prod_{1 \leq i<e}\left(\sigma^{i f}\left(\varpi_{E}\right)-\varpi_{E}\right)
\end{aligned}
$$

and

$$
\begin{aligned}
\sigma\left(b_{d}\right) & =\prod_{0 \leq i<e-1}\left(\sigma^{(i+1) f}\left(\varpi_{E}\right)-\varpi_{E}\right)^{-1} \cdot \prod_{1 \leq i<f}\left(\sigma^{i}(\zeta)-\zeta\right)^{-1} \\
& =\nu \prod_{1 \leq i<e}\left(\sigma^{i f}\left(\varpi_{E}\right)-\varpi_{E}\right)^{-1}
\end{aligned}
$$

we have

$$
\prod_{2 \leq i \leq d} a_{1, i} \cdot \sigma\left(b_{d}\right)=1=b_{1}
$$

Thus, we obtain

$$
m\left(\sigma_{T}\right) \sigma\left(h^{-1}\right)=h^{-1},
$$

so that the 1-cocycle $\lambda\left(\mathbf{T}_{\mathrm{sc}}\right)$ as in [15, Section 2.3] is trivial. This yields the lemma. 
Proposition 9.5. If e and $f$ are odd, then we have

$$
\Delta(\gamma, \iota(\gamma))=\lambda(E / F, \psi)^{-1} \cdot \Delta_{\mathrm{IV}}(\gamma, \iota(\gamma)) .
$$

If $e$ is odd and $f$ is even, then we have

$$
\Delta(\gamma, \iota(\gamma))=\lambda(E / F, \psi)^{-1} \cdot \eta_{E}\left(\gamma-\sigma_{+}(\gamma)\right) \cdot \Delta_{\mathrm{IV}}(\gamma, \iota(\gamma))
$$

If $e$ is even and $f$ is odd, then we have

$$
\Delta(\gamma, \iota(\gamma))=\lambda(E / F, \psi)^{-1} \cdot \omega(-1)^{e(f-1) / 4} \cdot \omega_{+}\left(\frac{\gamma-\sigma_{+}(\gamma)}{2 \varpi_{E}^{-1}}\right) \cdot \Delta_{\mathrm{IV}}(\gamma, \iota(\gamma))
$$

If $e$ and $f$ are even, then we have

$$
\Delta(\gamma, \iota(\gamma))=\lambda(E / F, \psi)^{-1} \cdot \omega_{+}\left(\frac{\gamma-\sigma_{+}(\gamma)}{2 \varpi_{E}^{-1}\left(\sigma^{f / 2}(\zeta)-\zeta\right)}\right) \cdot \Delta_{\mathrm{IV}}(\gamma, \iota(\gamma))
$$

Proof. Let $V$ be the virtual representation of $\Gamma$ given by (1.1). Then we have

$$
\varepsilon(V, \psi)=\lambda(E / F, \psi)^{-1}
$$

Hence, the proposition follows from (9.2) and Lemmas 9.3 and 9.4.

\section{§10. Proof of Theorem 1.4}

In this section, we finish the proof of Theorem 1.4. We may keep the assumption of Section 6 .

We fix a primitive $\left(q^{f}-1\right)$ th root of unity $\zeta$ in $E$ and choose $\varpi_{E}, \varpi_{F}$ as in Section 9. Let $\iota: \mathbf{H} \rightarrow \mathbf{G}$ be the embedding defined in Section 9 .

Let $\gamma \in H$ be a $G$-regular semisimple element such that $\operatorname{ord}_{E} \gamma=-1$. As in Section 8, we write $\gamma=\delta \gamma^{\prime}$ so that $\delta$ is $F(\delta) / F$-cuspidal and $\gamma^{\prime} \in 1+\mathfrak{p}_{E}$. Put $F^{\prime}=F(\delta)$, and put $\delta^{\prime}=\varpi_{E} \delta$. By Lemma $8.2, E / F^{\prime}$ is unramified and $\delta^{\prime} \in \mathfrak{o}_{E}^{\times}$. We may assume that $\delta^{\prime}$ is a root of unity in $E$.

Let $\mathbf{G}^{\prime}=\operatorname{Res}_{F^{\prime} / F} \mathrm{GL}_{d^{\prime}}$, where $d^{\prime}=d / e f^{\prime}$ and $f^{\prime}$ is the residual degree of $F^{\prime} / F$. Let $\iota^{\prime}: \mathbf{H} \rightarrow \mathbf{G}^{\prime}$ be the embedding as in Section 9. By Proposition 9.5, we have

$$
\Delta_{G^{\prime}, H}\left(\gamma^{\prime}, \iota^{\prime}\left(\gamma^{\prime}\right)\right)=\left\{\begin{array}{l}
\Delta_{G^{\prime}, H, \mathrm{IV}}\left(\gamma^{\prime}, \iota^{\prime}\left(\gamma^{\prime}\right)\right) \\
\quad \text { if } d^{\prime} \text { is odd }, \\
\eta_{E}\left(\gamma^{\prime}-\sigma_{+}\left(\gamma^{\prime}\right)\right) \cdot \Delta_{G^{\prime}, H, \mathrm{IV}}\left(\gamma^{\prime}, \iota^{\prime}\left(\gamma^{\prime}\right)\right) \\
\text { if } d^{\prime} \text { is even. }
\end{array}\right.
$$

Note that $\gamma^{\prime}$ is $G^{\prime}$-regular.

Let $F^{\prime \prime} \subset E$ be the unramified extension of $F$ of degree $f$. Then $\delta^{\prime} \in F^{\prime \prime}$. 
Lemma 10.1. Assume that $e$ is odd and that $f$ is even. Then $d^{\prime}$ is even if and only if $\delta^{\prime} \in E_{+}$.

Proof. Since $E / F$ is cyclic, $d^{\prime}$ is even if and only if $F^{\prime} \subset E_{+}$. Since $F^{\prime}=$ $F(\delta)$ and $\varpi_{E} \in E_{+}$, the assertion follows.

Lemma 10.2. Assume that $e$ is even. Then $d^{\prime}$ is odd and $\delta^{\prime} \in E_{+}$.

Proof. We have $F^{\prime \prime} \subset E_{+}$since $e$ is even. Hence, $E / E_{+}$is ramified, so that $d^{\prime}$ must be odd since $E / F^{\prime}$ is unramified.

LEMma 10.3. We have

$$
\begin{aligned}
\Delta_{G, H}(\gamma, \iota(\gamma))= & \lambda(E / F, \psi)^{-1} \cdot \Delta_{G^{\prime}, H}\left(\gamma^{\prime}, \iota\left(\gamma^{\prime}\right)\right) \\
& \times \begin{cases}1 & \text { if } e \text { and } f \text { are odd, } \\
\omega\left(\varpi_{F}\right)^{d / 2} & \text { if } e \text { is odd and } f \text { is even, } \\
\omega(-1)^{e(f-1) / 4} \cdot \omega_{+}\left(\delta^{\prime}\right) & \text { if } e \text { is even and } f \text { is odd, } \\
\omega_{+}\left(\sigma^{f / 2}(\zeta)-\zeta\right) \cdot \omega_{+}\left(\delta^{\prime}\right) & \text { if } e \text { and } f \text { are even. }\end{cases}
\end{aligned}
$$

Proof. By [19, lemme VII.3], we have

$$
\Delta_{G, H, \mathrm{IV}}(\gamma, \iota(\gamma))=\Delta_{G^{\prime}, H, \mathrm{IV}}\left(\gamma^{\prime}, \iota^{\prime}\left(\gamma^{\prime}\right)\right) .
$$

By Proposition 9.5, (10.1), and Lemmas 10.1 and 10.2, it suffices to show that

$$
\begin{aligned}
\eta_{E}\left(\gamma-\sigma_{+}(\gamma)\right)= & \omega\left(\varpi_{F}\right)^{d / 2} \\
& \times \begin{cases}\eta_{E}\left(\gamma^{\prime}-\sigma_{+}\left(\gamma^{\prime}\right)\right) & \text { if } e \text { is odd, } f \text { is even, and } \delta^{\prime} \in E_{+}, \\
1 & \text { if } e \text { is odd, } f \text { is even, and } \delta^{\prime} \notin E_{+}\end{cases}
\end{aligned}
$$

and that

$$
\omega_{+}\left(\frac{\gamma-\sigma_{+}(\gamma)}{2 \varpi_{E}^{-1}}\right)=\omega_{+}\left(\delta^{\prime}\right)
$$

if $e$ is even.

We first assume that $e$ is odd and $f$ is even. Then we have

$$
\eta_{E}\left(\gamma-\sigma_{+}(\gamma)\right)=\eta_{E}\left(\varpi_{E}^{-1}\right) \cdot \eta_{E}\left(\delta^{\prime} \gamma^{\prime}-\sigma_{+}\left(\delta^{\prime} \gamma^{\prime}\right)\right) .
$$

Since $\eta_{E}^{2}=\mathbb{1}$ and $e$ is odd, we have

$$
\eta_{E}\left(\varpi_{E}^{-1}\right)=\eta_{E}\left(\varpi_{E}\right)^{e}=\eta_{E}\left(\varpi_{F} \zeta^{a}\right)
$$


Since $\eta_{E}$ is unramified, we have $\eta_{E}\left(\zeta^{a}\right)=1$. Hence, we have

$$
\eta_{E}\left(\varpi_{E}^{-1}\right)=\eta_{E}\left(\varpi_{F}\right)=\omega\left(\mathrm{N}_{E_{+} / F}\left(\varpi_{F}\right)\right)=\omega\left(\varpi_{F}^{d / 2}\right) .
$$

If $\delta^{\prime} \in E_{+}$, then we have

$$
\eta_{E}\left(\delta^{\prime} \gamma^{\prime}-\sigma_{+}\left(\delta^{\prime} \gamma^{\prime}\right)\right)=\eta_{E}\left(\delta^{\prime}\right) \cdot \eta_{E}\left(\gamma^{\prime}-\sigma_{+}\left(\gamma^{\prime}\right)\right)=\eta_{E}\left(\gamma^{\prime}-\sigma_{+}\left(\gamma^{\prime}\right)\right)
$$

If $\delta^{\prime} \notin E_{+}$, then $\sigma_{+}\left(\delta^{\prime}\right) \neq \delta^{\prime}$, so that

$$
\delta^{\prime} \gamma^{\prime}-\sigma_{+}\left(\delta^{\prime} \gamma^{\prime}\right) \equiv \delta^{\prime}-\sigma_{+}\left(\delta^{\prime}\right) \not \equiv 0 \quad \bmod \mathfrak{p}_{E}
$$

Hence, we have $\delta^{\prime} \gamma^{\prime}-\sigma_{+}\left(\delta^{\prime} \gamma^{\prime}\right) \in \mathfrak{o}_{E}^{\times}$, so that $\eta_{E}\left(\delta^{\prime} \gamma^{\prime}-\sigma_{+}\left(\delta^{\prime} \gamma^{\prime}\right)\right)=1$.

We next assume that $e$ is even. Then we have

$$
\frac{\gamma-\sigma_{+}(\gamma)}{2 \varpi_{E}^{-1}}=\frac{\varpi_{E}^{-1} \delta^{\prime}\left(\gamma^{\prime}+\sigma_{+}\left(\gamma^{\prime}\right)\right)}{2 \varpi_{E}^{-1}}=\frac{\delta^{\prime}\left(\gamma^{\prime}+\sigma_{+}\left(\gamma^{\prime}\right)\right)}{2}
$$

Since $E / F$ is tamely ramified and $e$ is even, we have $2 \nmid q$, so that $\left(\gamma^{\prime}+\right.$ $\left.\sigma_{+}\left(\gamma^{\prime}\right)\right) / 2 \in 1+\mathfrak{p}_{E_{+}}$. Hence, we have

$$
\omega_{+}\left(\frac{\gamma^{\prime}+\sigma_{+}\left(\gamma^{\prime}\right)}{2}\right)=1
$$

Let $f^{G} \in C_{c}^{\infty}(G)$ be as in Section 7. By [19] and [20], there exists $f^{H} \in$ $C_{c}^{\infty}(H)$ such that $f^{G}$ and $f^{H}$ have matching orbital integrals. Then $f^{H}$ is determined by

$$
f^{H}(\gamma)=\Delta_{G, H}(\gamma, \iota(\gamma)) \cdot J^{\omega}\left(\gamma, f^{G}\right)
$$

for $G$-regular semisimple elements $\gamma \in H$. By Lemma 8.1, $f^{H}(\gamma)=0$ unless $\operatorname{ord}_{E} \gamma=-1$.

LEMMA 10.4. Let $\gamma \in H$ be a $G$-regular semisimple element such that that $\operatorname{ord}_{E} \gamma=-1$. Then we have

$$
f^{H}(\gamma)=\lambda(E / F, \psi)^{-1} \times \begin{cases}1 & \text { if } e \text { and } f \text { are odd, } \\ \omega\left(\varpi_{F}\right)^{d / 2} & \text { if } e \text { is odd and } f \text { is even }, \\ \omega(-1)^{e(f-1) / 4} & \text { if } e \text { is even and } f \text { is odd }, \\ \omega_{+}\left(\sigma^{f / 2}(\zeta)-\zeta\right) & \text { if } e \text { and } f \text { are even } .\end{cases}
$$


Proof. By the definition of $\iota$, we have

$$
\iota\left(\varpi_{E}\right)=\eta \cdot\left(\begin{array}{cc}
g_{1,1} & 0 \\
0 & \mathbf{1}_{d-f}
\end{array}\right)
$$

with some $g_{1,1} \in \mathrm{GL}_{f}\left(\mathfrak{o}_{F}\right)$ and

$$
\iota\left(\delta^{\prime}\right)=\operatorname{diag}\left(g^{\prime}, g^{\prime}, \ldots, g^{\prime}\right)
$$

with some $g^{\prime} \in \operatorname{GL}_{f}\left(\mathfrak{o}_{F}\right)$ such that $\operatorname{det} g^{\prime}=\mathrm{N}_{F^{\prime \prime} / F}\left(\delta^{\prime}\right)$. Hence, we have

$$
f^{G}(\delta)=f^{G}\left(\varpi_{E}^{-1} \delta^{\prime}\right)=\operatorname{vol}(I)^{-1} \cdot \omega\left(\mathrm{N}_{F^{\prime \prime} / F}\left(\delta^{\prime}\right)\right)^{-e(e+1) / 2} .
$$

If $e$ is odd, then we have

$$
\omega\left(\mathrm{N}_{F^{\prime \prime} / F}\left(\delta^{\prime}\right)\right)^{-e(e+1) / 2}=\omega^{e}\left(\mathrm{~N}_{F^{\prime \prime} / F}\left(\delta^{\prime}\right)\right)^{-(e+1) / 2}=1 .
$$

If $e$ is even, then we have

$$
\omega\left(\mathrm{N}_{F^{\prime \prime} / F}\left(\delta^{\prime}\right)\right)^{-e(e+1) / 2}=\omega\left(\mathrm{N}_{E_{+} / F}\left(\delta^{\prime}\right)\right)^{-(e+1)}=\omega_{+}\left(\delta^{\prime}\right)^{-(e+1)}=\omega_{+}\left(\delta^{\prime}\right) .
$$

By Lemmas 8.5 and 10.3, we have

$$
\begin{aligned}
& \Delta_{G, H}(\gamma, \iota(\gamma)) \cdot J^{\omega}\left(\gamma, f^{G}\right) \\
& =\lambda(E / F, \psi)^{-1} \cdot \Delta_{G^{\prime}, H}\left(\gamma^{\prime}, \iota^{\prime}\left(\gamma^{\prime}\right)\right) \cdot J^{\omega^{\prime}}\left(\gamma^{\prime}, \mathbf{1}_{K^{\prime}}\right) \\
& \quad \times \begin{cases}1 & \text { if } e \text { and } f \text { are odd, } \\
\omega\left(\varpi_{F}\right)^{d / 2} & \text { if } e \text { is odd and } f \text { is even, } \\
\omega(-1)^{e(f-1) / 4} & \text { if } e \text { is even and } f \text { is odd, } \\
\omega_{+}\left(\sigma^{f / 2}(\zeta)-\zeta\right) & \text { if } e \text { and } f \text { are even. }\end{cases}
\end{aligned}
$$

By the fundamental lemma [19, théorème VIII.5], we have

$$
\Delta_{G^{\prime}, H}\left(\gamma^{\prime}, \iota^{\prime}\left(\gamma^{\prime}\right)\right) \cdot J^{\omega^{\prime}}\left(\gamma^{\prime}, \mathbf{1}_{K^{\prime}}\right)=1
$$

This completes the proof.

Thus, we obtain

$$
J\left(\pi_{H}, f^{H}\right)=\lambda(E / F, \psi)^{-1} \times \begin{cases}1 & \text { if } e \text { and } f \text { are odd, } \\ \omega\left(\varpi_{F}\right)^{d / 2} & \text { if } e \text { is odd and } f \text { is even, } \\ \omega(-1)^{e(f-1) / 4} & \text { if } e \text { is even and } f \text { is odd, } \\ \omega_{+}\left(\sigma^{f / 2}(\zeta)-\zeta\right) & \text { if } e \text { and } f \text { are even, }\end{cases}
$$


where $\pi_{H}=\mathbb{1}$. By Proposition 7.8 and Lemma 10.5 below, we have

$$
J^{\omega}\left(\pi, f^{G}\right)=J\left(\pi_{H}, f^{H}\right)
$$

Hence, we have $c=1$.

To finish the proof of Theorem 1.4, it remains to prove the following lemma.

LEMma 10.5. Assume that $e$ and $f$ are even. Then we have

$$
\omega(-1)^{e f / 4} \cdot \omega\left(\varpi_{F}\right)^{d / 2}=\omega_{+}\left(\sigma^{f / 2}(\zeta)-\zeta\right)
$$

Proof. Note that $2 \nmid q$ since $E / F$ is tamely ramified and $e$ is even. By Lemma 10.6 below, we have $\varpi_{E}^{e}=\varpi_{F} \zeta^{a}$ with an odd integer $a$.

We first show that

$$
\omega\left(\varpi_{F}\right)^{d / 2}=-1
$$

The quadratic character $\omega_{+}=\omega \circ \mathrm{N}_{E_{+} / F}$ of $E_{+}^{\times}$associated to $E / E_{+}$satisfies the following:

- $\omega_{+}(\zeta)=-1$,

- $\omega_{+}\left(-\varpi_{E}^{2}\right)=1$,

- $\omega_{+}$is trivial on $1+\mathfrak{p}_{E_{+}}$.

Note that $F^{\prime \prime} \subset E_{+}$and $\sigma_{+}\left(\varpi_{E}\right)=-\varpi_{E}$. Hence, we have

$$
\begin{aligned}
\omega\left(\varpi_{F}\right)^{d / 2} & =\omega_{+}\left(\varpi_{F}\right) \\
& =\omega_{+}\left(\varpi_{E}^{e} \zeta^{-a}\right) \\
& =\omega_{+}\left((-1)^{e / 2} \cdot\left(-\varpi_{E}^{2}\right)^{e / 2} \cdot \zeta^{-a}\right) \\
& =\omega_{+}(-1)^{e / 2} \cdot \omega_{+}(\zeta)^{-a} \\
& =\omega(-1)^{e d / 4} \cdot(-1)^{-a} \\
& =-1 .
\end{aligned}
$$

We next show that

$$
\omega(-1)^{e f / 4}= \begin{cases}1 & \text { if } q \equiv 1 \bmod 4 \\ 1 & \text { if } q \equiv 3 \bmod 4 \text { and } f \equiv 0 \bmod 4 \\ -1 & \text { if } q \equiv 3 \bmod 4 \text { and } f \equiv 2 \bmod 4\end{cases}
$$


Note that, if $q \equiv 3 \bmod 4$, then $e \equiv 2 \bmod 4$ since $e \mid(q-1)$. Assume that $q \equiv 1 \bmod 4$. Then a primitive fourth root of unity $\zeta^{\left(q^{f}-1\right) / 4}$ belongs to $F$. Since $(-1)^{e f / 4}=\left(\zeta^{\left(q^{f}-1\right) / 4}\right)^{d / 2}$, we have

$$
\omega(-1)^{e f / 4}=\omega_{+}\left(\zeta^{\left(q^{f}-1\right) / 4}\right)=(-1)^{\left(q^{f}-1\right) / 4} .
$$

Since $f$ is even, $\left(q^{f}-1\right) / 4$ is even, so that $\omega(-1)^{e f / 4}=1$. Assume that $q \equiv 3 \bmod 4$. It suffices to show that $\omega(-1)=-1$. Let $\zeta^{\prime}=\zeta^{\left(q^{f}-1\right) /(q-1)}$ be a primitive $(q-1)$ th root of unity in $F$. Then $\omega\left(\zeta^{\prime}\right)$ is a primitive $e$ th root of unity in $\mathbb{C}^{\times}$since the restriction of $\omega$ to $\mathfrak{o}_{F}^{\times}$is of order $e$. Since $e \mid(q-1)$ and $(q-1) / 2$ is odd, we have

$$
\frac{q-1}{2} \equiv \frac{e}{2} \quad \bmod e .
$$

Hence, we have

$$
\omega(-1)=\omega\left(\zeta^{\prime(q-1) / 2}\right)=\omega\left(\zeta^{\prime}\right)^{e / 2}=-1
$$

We finally show that

$$
\omega_{+}\left(\sigma^{f / 2}(\zeta)-\zeta\right)= \begin{cases}-1 & \text { if } q \equiv 1 \bmod 4 \\ -1 & \text { if } q \equiv 3 \bmod 4 \text { and } f \equiv 0 \bmod 4 \\ 1 & \text { if } q \equiv 3 \bmod 4 \text { and } f \equiv 2 \bmod 4\end{cases}
$$

Put $\beta=\sigma^{f / 2}(\zeta)-\zeta \in \mathfrak{o}_{F^{\prime \prime}}^{\times}$. Then we have $\sigma^{f / 2}(\beta)=-\beta$, and hence $\left(\beta^{2}\right)^{q^{f / 2}-1} \equiv 1 \bmod \mathfrak{p}_{F^{\prime \prime}}$. Let $r$ be the order of $\beta^{2}$ in $\left(\mathfrak{o}_{F^{\prime \prime}} / \mathfrak{p}_{F^{\prime \prime}}\right)^{\times}$. Then we have $r \mid\left(q^{f / 2}-1\right)$. Since $2 \nmid q$, we have $\sigma^{f / 2}(\beta) \not \equiv \beta \bmod \mathfrak{p}_{F^{\prime \prime}}$. Hence, $2 r$ is the order of $\beta$ in $\left(\mathfrak{o}_{F^{\prime \prime}} / \mathfrak{p}_{F^{\prime \prime}}\right)^{\times}$and $2 r \nmid\left(q^{f / 2}-1\right)$. Let $b$ be an integer such that $(b, 2 r)=1$ and $\beta \equiv \zeta^{b\left(q^{f}-1\right) / 2 r} \bmod \mathfrak{p}_{F^{\prime \prime}}$. Then we have

$$
\omega_{+}(\beta)=\omega_{+}\left(\zeta^{b\left(q^{f}-1\right) / 2 r}\right)=(-1)^{b\left(q^{f}-1\right) / 2 r}=(-1)^{\left(q^{f}-1\right) / 2 r}=(-1)^{\left(q^{f / 2}+1\right) / 2} .
$$

Note that $b,\left(q^{f / 2}-1\right) / r$ are odd and

$$
\frac{q^{f}-1}{2 r}=\frac{q^{f / 2}-1}{r} \cdot \frac{q^{f / 2}+1}{2} .
$$

Hence, $\omega_{+}(\beta)=1$ if and only if $4 \mid\left(q^{f / 2}+1\right)$. This yields the assertion. 
LEMma 10.6. Let $F$ be a nonarchimedean local field of characteristic zero. Let $f$ be a positive integer. We fix a primitive $\left(q^{f}-1\right)$ th root of unity $\zeta$ in $\bar{F}$. Let $F^{\prime \prime}=F(\zeta)$ be the unramified extension of $F$ of degree $f$. Let e be a positive integer such that $e \mid(q-1)$.

Let $\varpi_{E}$ be a root of

$$
X^{e}-\varpi_{F} \zeta^{a},
$$

where $\varpi_{F}$ is a uniformizer of $F$ and $a$ is an integer such that

$$
\left(a, \frac{q^{f}-1}{q-1}, e\right)=1 .
$$

Then $E=F^{\prime \prime}\left(\varpi_{E}\right)$ is a tamely ramified cyclic extension of $F$ with ramification index e and residual degree $f$. Conversely, any such $E$ is of this form.

Proof. If $E$ is a tamely ramified cyclic extension of $F$ with ramification index $e$ and residual degree $f$, then there exist a uniformizer $\varpi_{F}$ of $F$ and an integer $a$ such that $E=F^{\prime \prime}\left(\varpi_{E}\right)$, where $\varpi_{E}$ is a root of $X^{e}-\varpi_{F} \zeta^{a}$.

Conversely, let $E=F^{\prime \prime}\left(\varpi_{E}\right)$, where $\varpi_{E}$ is a root of $X^{e}-\varpi_{F} \zeta^{a}, \varpi_{F}$ is a uniformizer of $F$, and $a$ is an integer. For each $0 \leq i \leq e-1$, $\varpi_{E} \zeta^{a(q-1) / e+i\left(q^{f}-1\right) / e}$ is a root of $X^{e}-\varpi_{F} \zeta^{a q}$. Hence, there exists an automorphism $\sigma$ of $E$ over $F$ given by

$$
\begin{aligned}
\sigma(\zeta) & =\zeta^{q}, \\
\sigma\left(\varpi_{E}\right) & =\varpi_{E} \zeta^{a(q-1) / e+i\left(q^{f}-1\right) / e} .
\end{aligned}
$$

Note that any extension to $E$ of the Frobenius automorphism of $F^{\prime \prime}$ over $F$ is of this form.

Let $n$ be the order of $\sigma$. Since $\sigma$ is an extension of the Frobenius automorphism of $F^{\prime \prime}$ over $F$, we have $f \mid n$. We have

$$
\begin{aligned}
\sigma^{f}\left(\varpi_{E}\right) & =\varpi_{E}\left(\zeta^{a(q-1) / e+i\left(q^{f}-1\right) / e}\right)^{1+q+\cdots+q^{f-1}} \\
& =\varpi_{E}\left(\zeta^{a(q-1) / e+i\left(q^{f}-1\right) / e}\right)^{\left(q^{f}-1\right) /(q-1)} \\
& =\varpi_{E}\left(\zeta^{a+i\left(q^{f}-1\right) /(q-1)}\right)^{\left(q^{f}-1\right) / e} .
\end{aligned}
$$

We have $\left(\zeta^{a(q-1) / e+i\left(q^{f}-1\right) / e}\right)^{\left(q^{f}-1\right) /(q-1)} \in F^{\times}$since its order divides $q-1$. Hence, we have

$$
\sigma^{j f}\left(\varpi_{E}\right)=\varpi_{E}\left(\zeta^{a+i\left(q^{f}-1\right) /(q-1)}\right)^{j\left(q^{f}-1\right) / e}
$$


for all $j$, so that $n=e f$ if and only if

$$
\left(a+i \frac{q^{f}-1}{q-1}, e\right)=1 .
$$

Hence, there exists $0 \leq i \leq e-1$ such that $\sigma$ is of order $e f$ if and only if

$$
\left(a, \frac{q^{f}-1}{q-1}, e\right)=1 .
$$

This completes the proof.

This completes the proof of Theorem 1.4.

\section{REFERENCES}

[1] J. Arthur and L. Clozel, Simple Algebras, Base Change, and the Advanced Theory of the Trace Formula, Ann. of Math. Stud. 120, Princeton University Press, Princeton, NJ, 1989.

[2] E. Artin and J. Tate, Class Field Theory, AMS Chelsea Publishing, Providence, 2009.

[3] M. Harris and R. Taylor, The Geometry and Cohomology of Some Simple Shimura Varieties, Ann. of Math. Stud. 151, Princeton University Press, Princeton, NJ, 2001.

[4] G. Henniart, La conjecture de Langlands locale pour GL(3), Mém. Soc. Math. Fr. (N.S.) 11-12, Math. Soc. Fr., Paris, 1984.

[5] - On the local Langlands conjecture for $\mathrm{GL}(n)$ : The cyclic case, Ann. of Math. (2) 123 (1986), 145-203.

[6] — Induction automorphe pour $\mathrm{GL}(n, \mathbb{C})$, J. Funct. Anal. 258 (2010), 30823096.

[7] G. Henniart and R. Herb, Automorphic induction for GL(n) (over local nonArchimedean fields), Duke Math. J. 78 (1995), 131-192.

[8] G. Henniart and B. Lemaire, Intégrales orbitales tordues sur $\mathrm{GL}(n, F)$ et corps locaux proches: Applications, Canad. J. Math. 58 (2006), 1229-1267.

[9] - Formules de caractères pour l'induction automorphe, J. Reine Angew. Math. 645 (2010), 41-84.

[10] - Changement de base et induction automorphe pour $\mathrm{GL}_{n}$ en caractéristique non nulle, Mém. Soc. Math. Fr. (N.S.) 124, Math. Soc. Fr., Paris, 2011.

[11] K. Hiraga and H. Saito, On L-packets for inner forms of $\mathrm{SL}_{n}$, Mem. Amer. Math. Soc. 215 (2012), no. 1013.

[12] R. E. Howe, Tamely ramified supercuspidal representations of $\mathrm{Gl}_{n}$, Pacific J. Math. 73 (1977), 437-460.

[13] R. E. Kottwitz and D. Shelstad, Foundations of Twisted Endoscopy, Astérisque 255, Soc. Math. France, Paris, 1999.

[14] J.-P. Labesse and R. P. Langlands, L-indistinguishability for SL(2), Canad. J. Math. 31 (1979), 726-785.

[15] R. P. Langlands and D. Shelstad, On the definition of transfer factors, Math. Ann. 278 (1987), 219-271.

[16] F. Shahidi, On certain L-functions, Amer. J. Math. 103 (1981), 297-355. 
[17] , Fourier transforms of intertwining operators and Plancherel measures for GL(n), Amer. J. Math. 106 (1984), 67-111.

[18] J. Tate, "Number theoretic background" in Automorphic forms, representations and L-functions (Corvallis, Orea, 1977), Pt. 2, Proc. Sympos. Pure Math. 33, Amer. Math. Soc., Providence, 1979, 3-26.

[19] J.-L. Waldspurger, Sur les intégrales orbitales tordues pour les groupes linéaires: un lemme fondamental, Canad. J. Math. 43 (1991), 852-896.

[20] - Le lemme fondamental implique le transfert, Compos. Math. 105 (1997), $153-236$.

[21] - La conjecture locale de Gross-Prasad pour les représentations tempérées des groupes spéciaux orthogonaux, preprint, arXiv:0911.4568 [math.RT].

Kaoru Hiraga

Department of Mathematics

Kyoto University

Kyoto 606-8502

Japan

hiraga@math.kyoto-u.ac.jp

Atsushi Ichino

Department of Mathematics

Kyoto University

Kyoto 606-8502

Japan

ichino@math.kyoto-u.ac.jp 